\title{
The effect of rotation on linear and non-linear double-diffusive convection in a sparsely packed, porous medium
}

\author{
N. RUDRAIAII,* I. S. SIIIVAKUMARA* and R. FRILDRICII $\dagger$ \\ * UGC-DSA Centre in Fluid Mechanics, Department of Mathematics, Central College, \\ Bangalore University, Bangalore-560001, India \\ $\dagger$ Institute for Fluid Mechanics, Technical University of Munich, Munich, F.R.G.
}

(Received 31 July 1984 and in final form 17 December 1985)

\begin{abstract}
The linear and non-linear stability of rotating double-diffusive convection in a sparsely packed porous medium is investigated considering a non-Darcy equation. In the case of linear theory both marginal and overstable motions are discussed. In the former case it is shown that the effect of Taylor number and porous parameter is to make the system more stable. In the latter case, however, it is shown that the bottom-heavy solute gradient and rotation destabilize the system under certain conditions. By drawing the stability boundaries in the Rayleigh number plane it is shown that the effect of rotation and porous parameter is to decrease the region of instabilities. Using the theory of self-adjoint operator the variation of critical eigenvalue with physical and boundary parameters is studied. In the case of non-linear theory, both steady and unsteady cases have been considered. In the unsteady case the transient behaviour concerning the variation of Nusselt number with time has been investigated, by solving numerically a seventh-order Lorenz model using the Runge-Kutta-Gill method. Interesting results are obtained by comparing these results with those of the steady case. Finally, the effect of porous parameter on streamfunction, isotherms, isohalines and zonal velocity is studied.
\end{abstract}

\section{INTRODUCTION}

THE OBJECTIVE of this paper is to study doublediffusive convection in a porous medium in the presence of rotation because of its importance in chemical engineering, petroleum industry, bio-mechanics and geophysical problems [1]. Furthermore, this study also throws some light on the physical and mathematical understanding of the mechanism of heat and mass transfer in many practical problems.

In the absence of rotation, theoretical [2-4] and experimental [5] works have been carried out on double-diffusive convection in a porous medium and it has been shown that the simultaneous presence of two components with different diffusivities can give rise, as in the case of viscous flow $[6,7]$, to a whole range of new phenomena. In contrast, very little is known about the effect of rotation on double-diffusive convection in a porous medium. Recently Chakrabarti and Gupta [1] have studied this problem and predicted overstable motions in the case of infinitesimal disturbances and subcritical instabilities in the case of finite amplitude disturbances. However, their analysis says nothing about predicting some of the surprising results that emerge from the linear theory. Even their finite amplitude analysis, which is based on a perturbation technique proposed by Stuart [8] and by Malkus and Veronis [9], is restricted to predicting only the subcritical instabilities from the first non-trivial eigenvalue and gives no information about the prediction of the amount of heat and mass transfer. These aspects are discussed in detail in this paper.
We note that rotation affects the hydrodynamic stability only when the Ekman thickness (which defines the scale on which the frictional forces are comparable to the Coriolis force) is of the order of the pore size or smaller. If this situation is realized the assumption entering the Darcy law is no longer valid. Therefore, to realize the effect of rotation we have to use the non-Darcy equation (see $[10,11]$ ) involving convective acceleration and the usual viscous force. For this we have to consider a special type of porous medium of porosity (which is the ratio of the pore space to the whole volume of the porous medium [12]) close to unity in which the resistance offered to the flow by the elements in unit volume of a medium is sufficiently small so that the non-linear convective forces arising from the curvature of curvilinear flow through a porous medium may not be negligible. Furthermore, the effects of viscous stresses caused by the distortion of the fluid velocity in a porous medium should be taken into consideration in addition to the non-linear convective acceleration. The non-Darcy equation incorporating the inertial and viscous effects in addition to the Darcy resistance is reported in refs. $[10,11]$ and with rotation is discussed in ref. [13]. In this paper we use their non-Darcy equation to investigate the following :

(i) To understand the phenomena of bifurcation in the presence of rotation with different values of the physical parameters of the problem.

(ii) To study the effect of rotation on the region of salt-finger and diffusive instabilities in the case 


\section{NOMENCLATURE}

\section{Bi Biot number, $h d / K$}

$d$ depth of the fluid layer

g acceleration due to gravity

$h$ heat transfer coefficient

$H$ total heat transport

$J(f, g)$ Jacobian, $\partial(f, g) / \partial(x, z)$

$k^{2} \quad \pi^{2}\left(\alpha^{2}+1\right)$

$k_{\mathrm{p}} \quad$ permeability of a porous medium

$K$ thermal conductivity

$\mathrm{Nu}$ Nusselt number

$\mathrm{Nu}^{\mathrm{s}}$ solute Nusselt number

$p \quad$ frequency, $p_{\mathrm{r}}+\mathrm{i} p_{\mathrm{i}}$

$P_{1} \quad$ porous parameter

q velocity of the fluid, $(u, v, w)$

$R_{\mathrm{T}}$ thermal Rayleigh number

$R_{s} \quad$ solute Rayleigh number

$S$ solute concentration

$\Delta S$ concentration difference between lower and upper layers

$\mathrm{Sh}$ solute analogue of $\mathrm{Bi}$

$T$ temperature

$\Delta T$ temperature difference between lower and upper layers
$\mathrm{Ta}$ Taylor number

$t$ time coordinate

$u, w$ horizontal and vertical velocity components

$V$ vertical component of velocity induced by rotation

$x, z$ horizontal and vertical space coordinates.

$\begin{array}{cl}\text { Greek symbols } \\ \alpha & \text { wave number } \\ \alpha_{\mathrm{t}} & \text { thermal expansion coefficient } \\ \alpha_{\mathrm{s}} & \text { solute analogue of } \alpha_{\mathrm{t}} \\ \kappa & \text { thermal diffusivity } \\ \kappa_{\mathrm{s}} & \text { solute analogue of } \kappa \\ \nu & \text { kinematic viscosity } \\ \rho & \text { density of the fluid } \\ \sigma & \text { Prandtl number } \\ \tau & \text { ratio of diffusivities } \\ \psi & \text { streamfunction } \\ \Omega & \text { constant angular velocity } \\ \omega & \text { frequency, } \omega_{\mathrm{r}}+\mathrm{i} \omega_{i}\end{array}$

of infinitesimal disturbances. In particular it will be shown, as in viscous flow (see [14]), that under some conditions a double-diffusive, fluidsaturated, porous layer-stable in the absence of rotation-becomes unstable in the presence of rotation and the effect of bottom-heavy solute gradient is to destabilize the system. These aspects were not discussed in the work of Chakrabarti and Gupta [1].

(iii) The available literature on this topic depends on the assumption of perfect conducting boundaries which is valid only when the Biot number tends to infinity. In many practical problems cited above, it will be of interest to study the effect of the finite Biot number on the eigenvalue without going into the detailed numerical computation. Using the concept of self-adjoint operator (see [15]) we try to find the effect of Biot number, solute Rayleigh number, Taylor number and porous parameter on the monotonicity of critical Rayleigh number. Our object here is to show under what conditions the critical Rayleigh number for marginal convection is an upper bound for finite Biot number and to find an analytical relation for the variation of critical Rayleigh number with respect to solute Rayleigh number, Taylor number and porous parameter. This aspect has not been given any attention in the previous work on double-diffusive convection in a porous medium.

(iv) To study non-linear convection considering both steady and unsteady convective motions.

\section{FORMULATION OF THE PROBLEM}

Consider a sparsely packed, porous layer, saturated with Boussinesq fluid of infinite horizontal extent confined between parallel, stress-free boundaries at $z=0$ and $z=d$ at which the temperatures are $T_{0}$ and $T_{0}-\Delta T$, respectively. The layer rotates uniformly about the $z$-axis with a constant angular velocity $\Omega$. We denote one of the components by $\tilde{S}$ and suppose that the concentration of $\tilde{S}$ is held at $S_{0}$ and $S_{0}-\Delta S$ at the lower and upper boundaries, respectively. We write the total temperature and salinity as

$$
(\tilde{T}, \tilde{S})=\left(T_{0}, S_{0}\right)-(\Delta T, \Delta S) \frac{z}{d}+(\theta, \Sigma)(x, y, z, t)
$$

where $\Delta T, \Delta S>0$. In the Boussinesq approximation the density is taken to be $\rho=\rho_{0}\{1$ $\left.-\alpha_{1}\left(\tilde{T}-T_{0}\right)+\alpha_{3}\left(\tilde{S}-S_{0}\right)\right\}$, where $\rho_{0}$ is the density at temperature $T_{0}$ and concentration $S_{0}$ and $\alpha_{t}$, $\alpha_{3}>0$. We regard the porous medium as an assemblage of small, identical, spherical particles fixed in the space of porosity close to unity. In this case the required basic equations for a representative elementary volume, following refs. $[10,16]$, are:

$$
\begin{aligned}
& \frac{\partial \mathbf{q}}{\partial t}+(\mathbf{q} \cdot \nabla) \mathbf{q}+2 \Omega \hat{k} \times \mathbf{q}=-\frac{1}{\rho_{0}} \nabla p \\
&+\frac{\rho}{\rho_{0}} \mathbf{g}-\frac{v}{k_{\mathrm{p}}} \mathbf{q}+\nu \nabla^{2} \mathbf{q} \\
& M \frac{\partial \tilde{T}}{\partial t}+(\mathbf{q} \cdot \nabla) \tilde{T}=\kappa \nabla^{2} \tilde{T}
\end{aligned}
$$




$$
\begin{gathered}
\frac{\partial \tilde{S}}{\partial t}+(\mathbf{q} \cdot \nabla) \tilde{S}=\kappa_{s} \nabla^{2} \tilde{S} \\
\nabla \cdot \mathbf{q}=0
\end{gathered}
$$

where $\mathbf{q}=(u, v, w)$ is the mean filter velocity of the fluid, $p$ the pressure, $M=(\rho c)^{*} /(\rho c)_{\mathrm{f}}$ is the dimensionless heat capacity and $k_{\mathrm{p}}$ is the permeability of the porous medium. Since our interest here is a liquidsaturated, porous medium, the heat capacity ratio, $M$, can be assumed, with sufficient accuracy, to be unity. We note that (2) is similar to the equation given by Saffman [17] in the case of dusty gas theory. For simplicity of analysis, we confine ourselves to the twodimensional rolls, so that all the physical quantities are independent of $y$. We introduce a streamfunction $\psi$ such that

$$
u=\partial \psi / \partial z, \quad w=-\partial \psi / \partial x
$$

Eliminating the pressure from (2) and using the scales $v / d, d^{2} / \kappa$ and $d$ for velocity, time and distance, respectively, the system (2)-(4) may be written using (6), in dimensionless form, as :

$$
\begin{aligned}
& {\left[\frac{1}{\sigma} \frac{\partial}{\partial t}-\left(\nabla^{2}-P_{1}^{-1}\right)\right] \nabla^{2} \psi } \\
&-T a^{1 / 2} \frac{\partial V}{\partial z}=\frac{1}{\sigma}\left(\tau \frac{\partial \Sigma}{\partial x}-\frac{\partial \theta}{\partial x}\right)+J\left(\psi, \nabla^{2} \psi\right) \\
& {\left[\frac{1}{\sigma} \frac{\partial}{\partial t}-\left(\nabla^{2}-P_{1}^{-5}\right)\right] V=J(\psi, V)-T a^{1 / 2} \frac{\partial \psi}{\partial z} } \\
&\left(\frac{\partial}{\partial t}-\nabla^{2}\right) \theta=-\sigma R_{\mathrm{T}} \frac{\partial \psi}{\partial x}+\sigma J(\psi, \theta) \\
&\left(\frac{\partial}{\partial t}-\tau \nabla^{2}\right) \Sigma=-\sigma \frac{R_{3}}{\tau} \frac{\partial \psi}{\partial x}+\sigma J(\psi, \Sigma)
\end{aligned}
$$

where $\sigma=v / \kappa, \tau=\kappa_{\mathrm{s}} / \kappa, P_{1}=k_{\mathrm{p}} / d^{2}, R_{\mathrm{T}}=\alpha_{\mathrm{t}} g \Delta T d^{3} / v \kappa$, $R_{\mathrm{s}}=\alpha_{\mathrm{s}} g \Delta S d^{3} / v \kappa, T a=4 \Omega^{2} d^{4} / v^{2}$ and $V$ is the zonal velocity induced by rotation.

The boundary conditions are:

$$
\psi=\frac{\partial^{2} \psi}{\partial z^{2}}=\theta=\Sigma=\frac{\partial V}{\partial z}=0 \quad \text { at } \quad z=0,1 .
$$

\section{LINEAR STABIUITY ANALYSIS}

The criterion for the onset of convection is obtained from the linear stability problem by setting the Jacobian terms in $(7)-(10)$ to zero. To examine the stability of these equations we look for the solutions of the form

$$
\begin{gathered}
\psi \sim \mathrm{e}^{\omega t} \sin (\pi \alpha x) \sin (\pi z) \\
\theta, \Sigma \sim \mathrm{e}^{\omega t} \cos (\pi \alpha x) \sin (\pi z) .
\end{gathered}
$$

Substituting these into the linearized equations of (7)(10) and after some simplification we obtain the dispersion relation

$$
\begin{aligned}
\frac{p^{4}}{\sigma} & +\frac{p^{3}}{\sigma}[1+\tau+2 \sigma \eta] \\
& +p^{2}\left[\sigma \eta^{2}+\tau / \sigma+2 \eta(1+\tau)\right. \\
& \left.+R_{\mathrm{Ta}}^{\prime}-R_{\mathrm{T}}^{\prime}+R_{\mathrm{s}}^{\prime}\right]+p\left[\sigma \eta^{2}(1+\tau)\right. \\
& +2 \tau \eta+R_{\mathrm{Ta}}^{\prime}(1+\tau) \\
& \left.-R_{\mathrm{T}}^{\prime}(\tau+\sigma \eta)+R_{\mathrm{s}}^{\prime}(1+\sigma \eta)\right] \\
& +\tau \sigma \eta\left[\eta+\frac{R_{\mathrm{Ta}}^{\prime}}{\sigma \eta}-R_{\mathrm{T}}^{\prime}+\frac{R_{\mathrm{s}}^{\prime}}{\tau}\right]=0
\end{aligned}
$$

where

$$
\begin{gathered}
p=\frac{\omega}{k^{2}}, \quad R_{\mathrm{T}}^{\prime}=R_{\mathrm{T}} \frac{\pi^{2} \alpha^{2}}{k^{6}}, \\
R_{\mathrm{s}}^{\prime}=R_{\mathrm{s}} \frac{\pi^{2} \alpha^{2}}{k^{6}}, \quad R_{\mathrm{Ta}}^{\prime}=R_{\mathrm{Ta}} \frac{\pi^{2}}{k^{6}}, \\
R_{\mathrm{Ta}}=\sigma T a, \quad \eta=1+\frac{1}{P_{\mathrm{l}} k^{2}} \quad \text { and } \quad k^{2}=\pi^{2}\left(\alpha^{2}+1\right) .
\end{gathered}
$$

We note that this dispersion relation is of fourth order in the growth rate $\omega$, in contrast to the cubic equation obtained by Rudraiah et al. [3, 4] in the case of thermohaline convection in a porous medium in the absence of rotation. For certain strengths of rotation and concentration gradient the marginal state is valid depending on the values of $\sigma, P_{1}$ and $\tau$, and for other values overstable motions are preferred. Although Chakrabarti and Gupta [1] have studied the linear analysis, their work does not mention the marginal state, particularly, with regard to predicting the effects of $T a, R_{\mathrm{s}}, \tau$ and $P_{1}$ on cell pattern. These are discussed, separately, in this section.

\subsection{Marginal state}

There is a marginal state $\left(P_{\mathrm{r}}=0\right.$ and $\left.P_{\mathrm{i}}=0\right)$ when $R_{\mathrm{T}}=R_{\mathrm{T}}^{\mathrm{c}}$, where

$$
R_{\mathrm{T}}^{\mathrm{c}}=\frac{R_{\mathrm{s}}}{\tau}+\frac{\pi^{4}\left(\alpha^{2}+1\right)^{3}}{\alpha^{2}} \eta+\frac{T a}{\eta \alpha^{2}},
$$

provided $\alpha^{2}=x$, satisfies the equation

$$
\begin{gathered}
2 x^{5}+5\left(\eta^{\prime}+0.4\right) x^{4}+4 \eta^{\prime}\left(\eta^{\prime}+1\right) x^{3} \\
+\left\{\eta^{\prime}\left(\eta^{\prime 2}+2 \eta^{\prime}-1\right)-\frac{T a}{\pi^{4}}\right\} x^{2} \\
-\left\{\eta^{\prime 2}+\frac{T a}{\pi^{4}}\right\}\left(2 x+\eta^{\prime}\right)=0
\end{gathered}
$$

with

$$
\eta^{\prime}=1+\frac{1}{P_{1} \pi^{2}}
$$

The critical wavenumbers tabulated for different values of $T a$ and $P_{3}$ are shown in Table 1 . This table clearly depicts the combined effect of rotation and porous parameter on the critical wavenumber. We see 
Table 1. Critical wavenumbers for different values of $P_{1}$ and $T a$

\begin{tabular}{|c|c|c|c|c|c|c|c|c|}
\hline$P_{1}$ & $\begin{array}{c}T a=10 \\
\alpha_{c}^{2}\end{array}$ & $T a=\underset{\alpha_{a}^{2}}{=} 10^{2}$ & $T a=10^{3}$ & $\begin{array}{c}T a=10^{4} \\
\alpha_{\mathrm{c}}^{2}\end{array}$ & $T a=10^{5}$ & $T a=10^{6}$ & $T a=10^{7}$ & $\begin{array}{c}T a=10^{8} \\
\alpha_{\varepsilon}^{2}\end{array}$ \\
\hline$\infty$ & 0.5222 & 0.6815 & 1.3949 & 3.2896 & 7.5393 & 16.7648 & 36.6539 & 79.5491 \\
\hline $10^{-1}$ & 0.6265 & 0.6887 & 1.1155 & 2.7221 & 6.8163 & 15.9739 & 35.8507 & 78.7374 \\
\hline $10^{-2}$ & 0.8658 & 0.8689 & 0.9004 & 1.1750 & 2.7408 & 9.5254 & 28.6251 & 71.2861 \\
\hline $10^{-3}$ & 0.9812 & 0.9812 & 0.9817 & 0.9859 & 1.0282 & 1.3818 & 3.2956 & 13.5063 \\
\hline $10^{-4}$ & 0.9998 & 0.9980 & 0.9980 & 0.9981 & 0.9985 & 1.0030 & 1.0467 & 1.4109 \\
\hline $10^{-5}$ & 0.9998 & 0.9998 & 0.9998 & 0.9998 & 0.9998 & 0.9999 & 1.0003 & 1.0048 \\
\hline
\end{tabular}

that when the resistance offered to the flow by the elements in unit volume is sufficiently large (i.e. the usual Darcy law) the effect of rotation is negligible for values of $T a<10^{6}$. But for larger rotation rates we see that it, to some extent, overcomes the effect of porous parameter on the critical wavenumber. When $\eta^{\prime}=1$ (i.e. $P_{1} \rightarrow \infty$ ), (15) can be written as

$$
\left[2 x^{3}+3 x^{2}-\left(1+\frac{T a}{\pi^{4}}\right)\right](x+1)^{2}=0 .
$$

Since $(x+1)^{2} \neq 0$, it reduces to the viscous case discussed by Shivakumara et al. [15]. The plane surface of marginal stability on which the relation (14) holds will be denoted by $\mathscr{P}$.

\subsection{Overstable state}

For oscillatory modes $P_{\mathrm{r}}=0, P_{\mathrm{i}} \neq 0$, i.e. $P=\mathrm{i} P_{\mathrm{i}}$, where $P_{\mathrm{i}}$ has to be real. In this case we can rewrite (13) in the form

$$
\begin{aligned}
R_{\mathrm{T}}=\frac{\tau k^{4}+\omega_{i}^{2}}{\tau^{2} k^{4}+\omega_{i}^{2}} R_{\mathrm{s}}+\left[\eta k^{6}-k^{2} \frac{\omega_{i}^{2}}{\sigma}\right. \\
\left.\quad+\pi^{2} R_{\mathrm{Ta}} \frac{\left(\sigma \eta k^{4}+\omega_{i}^{2}\right)}{\left(\sigma^{2} \eta^{2} k^{4}+\omega_{i}^{2}\right)}\right] / \pi^{2} \alpha^{2}+\mathrm{i} \omega_{i} k^{2} N
\end{aligned}
$$

with

$$
N=\frac{R_{\mathrm{s}}(\tau-1)}{\tau^{2} k^{4}+\omega_{\mathrm{i}}^{2}}+\frac{\pi^{2} R_{\mathrm{Ta}}(\sigma \eta-1)}{\pi^{2} \alpha^{2}\left(\sigma^{2} \eta^{2} k^{4}+\omega_{i}^{7}\right)}+\frac{k^{2}(\sigma \eta+1)}{\pi^{2} \alpha^{2} \sigma} .
$$

The physical quantity $R_{\mathrm{T}}$ must be real, so that (16) implies that either $\omega_{\mathrm{i}}=0$ or $N=0$. For oscillatory $\left(\omega_{\mathrm{i}} \neq 0\right)$ neutral solutions, (16) requires $N=0$. The vanishing of $N$ provides a dispersion relation of the form

$$
\Delta_{1} \omega_{i}^{4}+\Delta_{2} \omega_{i}^{2}+\Delta_{3}=0
$$

where

$$
\begin{aligned}
& \Delta_{1}=k^{2}(\sigma \eta+1) \\
& \begin{aligned}
& \Delta_{2}=\pi^{2} \alpha^{2} \sigma(\tau-1) R_{\mathrm{s}}+\pi^{2} \sigma(\sigma \eta-1) R_{\mathrm{Ta}_{\mathrm{a}}} \\
&+ k^{6}(\sigma \eta+1)\left(\sigma^{2} \eta^{2}+\tau^{2}\right) \\
& \Delta_{3}=k^{4}\left[\pi^{2} \alpha^{2} \sigma^{3} \eta^{2}(\tau-1) R_{\mathrm{s}}\right.+\pi^{2} \tau^{2} \sigma(\sigma \eta-1) R_{\mathrm{Ta}} \\
&\left.+\tau^{2} \sigma^{2} \eta^{2}(\sigma \eta+1) k^{6}\right] .
\end{aligned}
\end{aligned}
$$

Since (18) is a quadratic in $\omega_{i}^{2}$, it can give rise to more than one positive value of $\omega_{\mathrm{i}}^{2}$ for fixed $\alpha^{7}, R_{s}, T a, \sigma$, $P_{1}$ and $\tau$. This has important implications for the linear stability of a rotating, doubly-diffusive fluid in a porous medium. Thus, it is worthwhile to establish necessary conditions for the existence of two oscillatory neutral solutions. From Descartes' rule of signs in order for (19) to have two positive roots, it is necessary that $\Delta_{2}<0$ and $\Delta_{3}>0$, from which it follows, using (20) and (21), that

$$
\begin{aligned}
\pi^{2} \sigma^{2}(\sigma \eta-1) T a+k^{6}(\sigma \eta+1)\left(\tau^{2}+\sigma^{2} \eta^{2}\right) & \\
<\pi^{2} \alpha^{2} \sigma(1-\tau) R_{\mathrm{s}}<\pi^{2} \tau^{2}(\sigma \eta-1) T a / \eta^{2} & +\tau^{2}(\sigma \eta+1) k^{6}
\end{aligned}
$$

Therefore

$0<k^{6}(\sigma \eta+1) \sigma^{2} \eta^{2}<\pi^{2} T a(\tau / \eta+\sigma)(\sigma \eta-1)(\tau / \eta-\sigma)$

which is equivalent to requiring that one of the conditions

$$
\tau<\sigma \eta<1
$$

or

$$
\tau>\sigma \eta>1
$$

be satisfied.

The results obtained for the combinations of values of $\tau, \sigma$ and $\eta$ are described below.

For $\tau<\sigma \eta\rangle 1$, neither (22a) nor (22b) is satisfied and no more than one oscillatory neutral solution can exist for each wavenumber $\alpha$, for the case $R_{\mathrm{s}}>0$. In this range, we have deduced some important information about the neutral curves in the $R_{\mathrm{T}}-\alpha^{2}$ plane by locating the branch points at which the steady and oscillatory neutral curves join. This will occur at wavenumbers for which $\omega_{i}=0$ is a root of (18). Thus $\Delta_{3}\left(\alpha_{b}^{2}\right)=0$, or equivalently

$$
\begin{aligned}
& (\sigma+1)\left(\alpha_{b}^{2}+1\right)^{5}+(3 \sigma+2) \gamma\left(\alpha_{b}^{2}+1\right)^{4}+\left[(3 \sigma+1) \gamma^{2}\right. \\
& \left.\quad+\sigma(\tau-1) R_{s} /\left(\pi^{4} \tau^{2}\right)\right]\left(\alpha_{b}^{2}+1\right)^{3}+\left[\sigma \gamma^{3}+\sigma(\tau-1)\right. \\
& \left.\quad \times(2 \gamma-1) R_{s} /\left(\pi^{4} \tau^{2}\right)+(\sigma-1) T a / \pi^{4}\right]\left(\alpha_{b}^{2}+1\right)^{2} \\
& \quad+\left[\sigma \gamma(\gamma-2)(\tau-1) R_{s} /\left(\pi^{4} \tau^{2}\right)+\sigma \gamma T a / \pi^{4}\right]\left(\alpha_{b}^{2}+1\right) \\
& \quad-\sigma \gamma^{2}(\tau-1) R_{s} /\left(\pi^{4} \tau^{2}\right)=0
\end{aligned}
$$

where

$$
\gamma=1 / P_{1} \pi^{2}
$$


This is a fifth-degree equation in $\alpha_{b}^{2}$ in contrast to the cubic equation familiar from the two-component, rotating fluid in the absence of a porous medium (see [14]). The branch points can be determined by solving (23) for fixed values of $T a, R_{s}, \sigma, \tau$ and $P_{1}$. The typical neutral curves in the $R_{\mathrm{T}}-\alpha^{2}$ plane are shown in Figs. $1(\mathrm{a})-(\mathrm{c})$ for two values of porous parameters. From these figures it is clear that the neutral curves are connected in a topoligical sense (refs. [14, 18]). This connectedness allows the linear stability criteria to be expressed in terms of a single 'critical thermal Rayleigh number', $\boldsymbol{R}_{\mathrm{T}}^{\mathrm{c}}$, below which the configuration is linearly stable and above which the system is definitely unstable. The numerical determination of $R_{\mathrm{T}}$ proceeds as follows. First determine the number of positive solutions of (23). If there are none, then no oscillatory instability is possible. If there are two, then the minimum (over $\alpha^{2}$ ) of (16) with $\omega_{i}^{2}$ given by (18) gives the value of $R_{\mathrm{T}}$.

When $\tau<\sigma \eta<1$, it is found that a rotating, doubly-diffusive porous layer can be destabilized by increasing $R_{\mathrm{s}}$, i.e. by adding heavy solute to the bottom. The destabilization manifests itsclf as a minimum in the $R_{\mathrm{T}}-R_{\mathrm{s}}$ plot, as shown in Figs. 2(a)-(c) for $P_{1}=10^{-2}, 10^{-3}$ and $10^{-4}$, respectively. From these figures it is evident that for certain combinations of $\sigma, \tau, P_{\mathrm{l}}$ and $T a$ there is some intermediate range of $R_{\mathrm{s}}$ in which the porous layer is destabilized by increasing $R_{\mathrm{s}}$ and stabilized by decreasing $R_{\mathrm{s}}$.

In the case of rotating viscous flow, Veronis [19] has given the physical explanation for the existence of subcritical instabilities for infinitesimal overstable motion and finite-amplitude steady motion based on the change in phase of dependent field quantities. Recently, Pearlstein [14], has given a different physical explanation, based on the concept developed by Acheson [20], for the curious behaviour that the addition of a suitably strong 'bottom-heavy' density gradient renders the system unstable, using the concept of frequency phenomena. The same analogy can be extended to the case of a porous medium with suitable change in porous parameter. For this purpose the graph of frequency against $R_{\mathrm{s}}$ is also drawn in Figs. 2 (a) - (c). These figures show that the destabilization is associated with a monotonically increasing $\omega_{i} \sigma / k^{2}$, which is a non-dimensional quantity and the range of destabilizing effect by $R_{\mathrm{s}}$ increases as $P_{1}$ decreases. This is because the diffusion of solute in a porous medium, for small values of $\tau$, is so slow that substantial changes in the bobbing frequency can be produced by changes in $R_{\mathrm{s}}$ that have little stabilizing effect via solute diffusion. Thus, the frequency can be 'tuned' by adjusting $R_{\mathrm{s}}$. If the frequency is too small, a bobbing parcel will always remain in approximatc thermal equilibrium with its environment. If the frequency is too high, no significant heat transfer will occur into or out of the parcel in the first place. In either extreme, the basic overstability mechanism is operating at less than optimal efficiency. At some intermediate frequency, however, the maximum efficiency is achieved, and overstable oscillations set in at a lower value of $R_{\mathrm{T}}$ than is possible for larger or smaller frequencies.
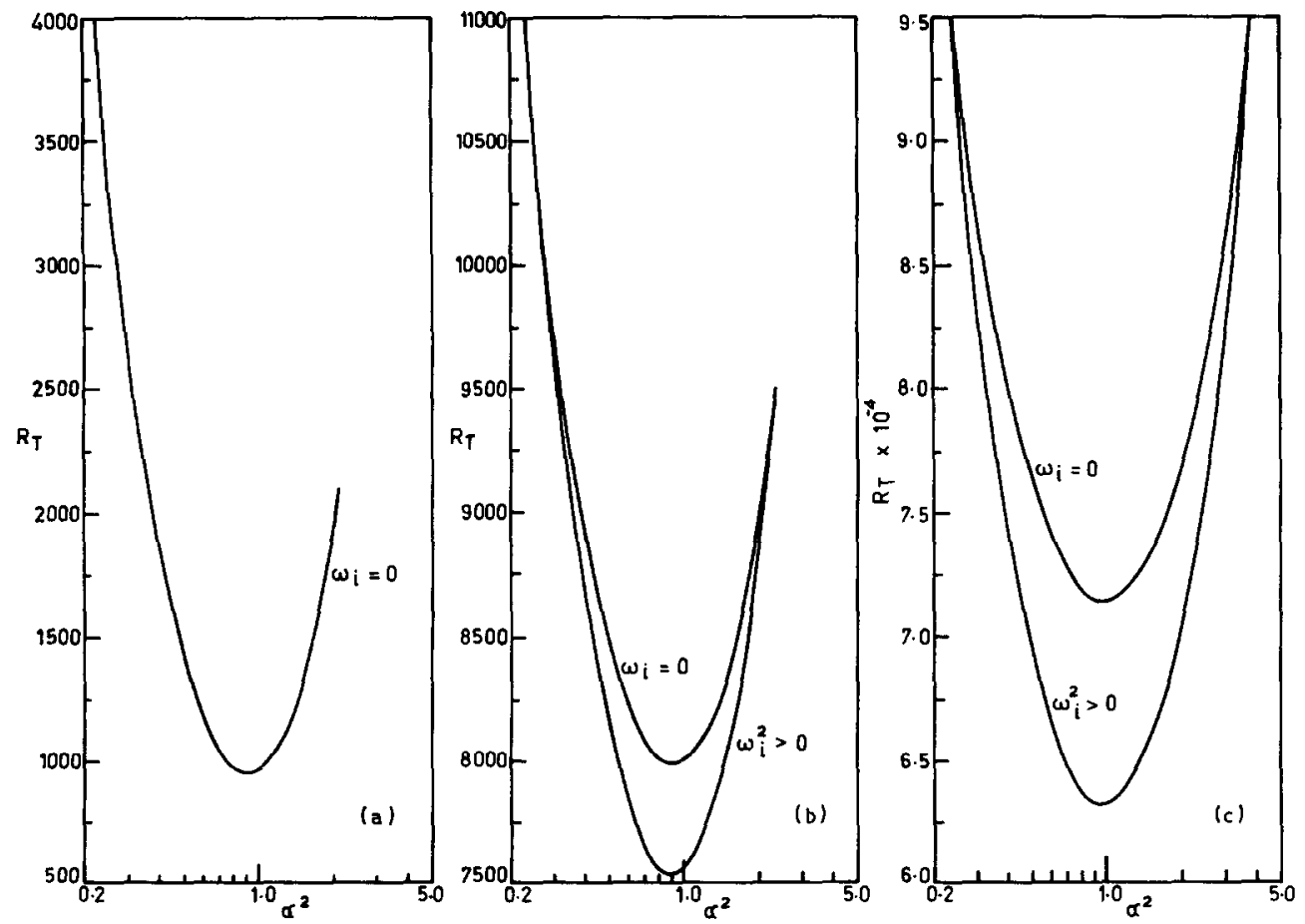

FIG. 1. Curves of neutral stability in the $R_{T} \alpha^{2}$ plane for (a) $\tau=0.32, \sigma=2.0 \mathrm{Ta}=10^{3}, P_{1}=10^{-2}$ and $R_{\mathrm{s}}=-1250$; (b) $\tau=0.32, \sigma=2.0, T a=10^{3}, P_{1}=10^{-2}$ and $R_{\mathrm{s}}=10^{3}$; (c) $\tau=0.32, \sigma=2.0, T a=10^{3}$, $P_{1}=10^{-3}$ and $R_{\mathrm{s}}=10^{4}$. 

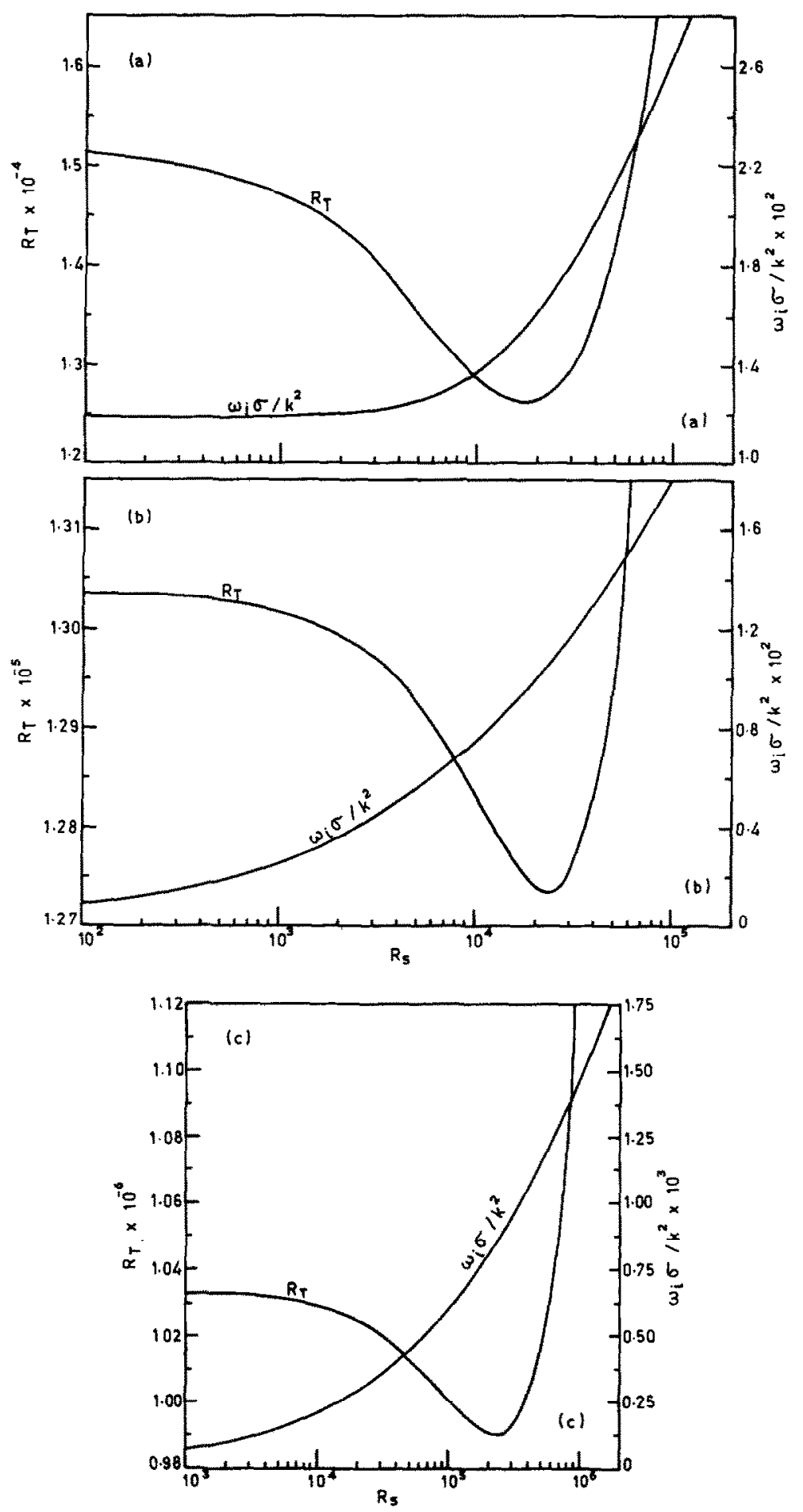

Fig. 2. Variation of $R_{\mathrm{T}}$ and $\omega_{i} \sigma / k^{2}$ with $R_{\mathrm{s}}$. (a) $\tau=0.003, \sigma=0.025, T a=10^{6}$ and $P_{1}=10^{-7}$; (b) $\tau=0.003$, $\sigma=0.025 T a=6 \times 10^{6}$ and $P_{1}=10^{-3}$; (c) $\tau=0.001, \sigma=0.002, T a=4 \times 10^{8}$ and $P_{1}=10^{-4}$.

This is consistent with the results shown in Fig. 2 for different values of $P_{1}$.

For combinations of $\tau, \sigma$ and $\eta$ such that $\tau>\sigma \eta<1$ neither (22a) nor (22b) is satisfied, so again no more than one oscillatory neutral solution per wavenumber is possible. Also (23) gives the number of branch points and the corresponding neutral curves are shown in Figs. 1(a)-(c). The neutral curves are again connected, so that the linear stability analysis need only provide a critical value of $R_{\mathrm{r}}$ and the associated 
critical wavenumber. A most striking feature observed is the crossing of the $T a=0$ and $T a=10^{5}$ loci for $P_{1}=10^{-2}, R_{\mathrm{s}}=4500, \sigma=0.025$ and $\tau=0.28$. For example, a non-rotating, porous layer stratified according to $R_{\mathrm{T}}=1.7 \times 10^{4}, R_{\mathrm{s}}=4500$ is predicted by linear theory to be stable, but when rotating with $T a=10^{5}$, it is unstable. The basic thermal and compositional stratifications are identical in the presence and absence of rotation so that this apparent destabilization is not produced by a rotation-induced density rearrangement, such as by baro-diffusion. Similar results were obtained by Pearlstein [14] for a rotating, double-diffusive fluid layer in a viscous flow. This destabilization appears as a minimum in the $R_{\mathrm{T}}-T a$ curve, as shown in Fig. 3. From this figure it is also clear that the destabilization is associated with an increase in $\omega_{i} \sigma / k^{2}$ and as $\omega_{i} \sigma / k^{2}$ goes on increasing, rotation again serves to stabilize the porous layer. The existence of such a minimum suggests that this destabilization by rotation may have a physical basis similar to that of destabilizing effect of a 'stable" density gradient in certain convection problems, including the present problem when $\tau<\sigma \eta<1$. This can bc explained as follows. For small values of $T a$, the frequency $\omega_{\mathrm{i}}$ is relatively small so that the parcel can remain in approximate density equilibrium with its environment, via the diffusion of heat and solute. As $\mathrm{Ta}$ increases, however, so does the oscillation frequency. This has the effect of making it more difficult for the parcel to remain in density equilibrium with its surroundings as it bobs up and down, and the oscillations will grow. Of course, if $\omega_{i}$ becomes too large, the basic overstability mechanism will fail because very little heat or solute will be transferred to the parcel during a cycle. Thus, we see how instability can be facilitated by an increase in $\omega_{i}$, and that if $\omega_{i}$ is too small or too large, the basic overstability mechanism is not very efficient. Again, as explained before, for some intermediate value of $\omega_{i}$, the overstability mechanism achieves its optimal efficiency, and convection becomes possible at a lower value of $R_{\mathrm{T}}$ than is possible for smaller or larger values of $\omega_{\mathrm{i}}$, This is consistent with the results shown in Fig. 3 for two values of $P_{1}$. Comparing Figs. $3(\mathrm{a})$ and (b) we found that the range of destabilizing effect by $T a$ increases as $P_{1}$ decreases.
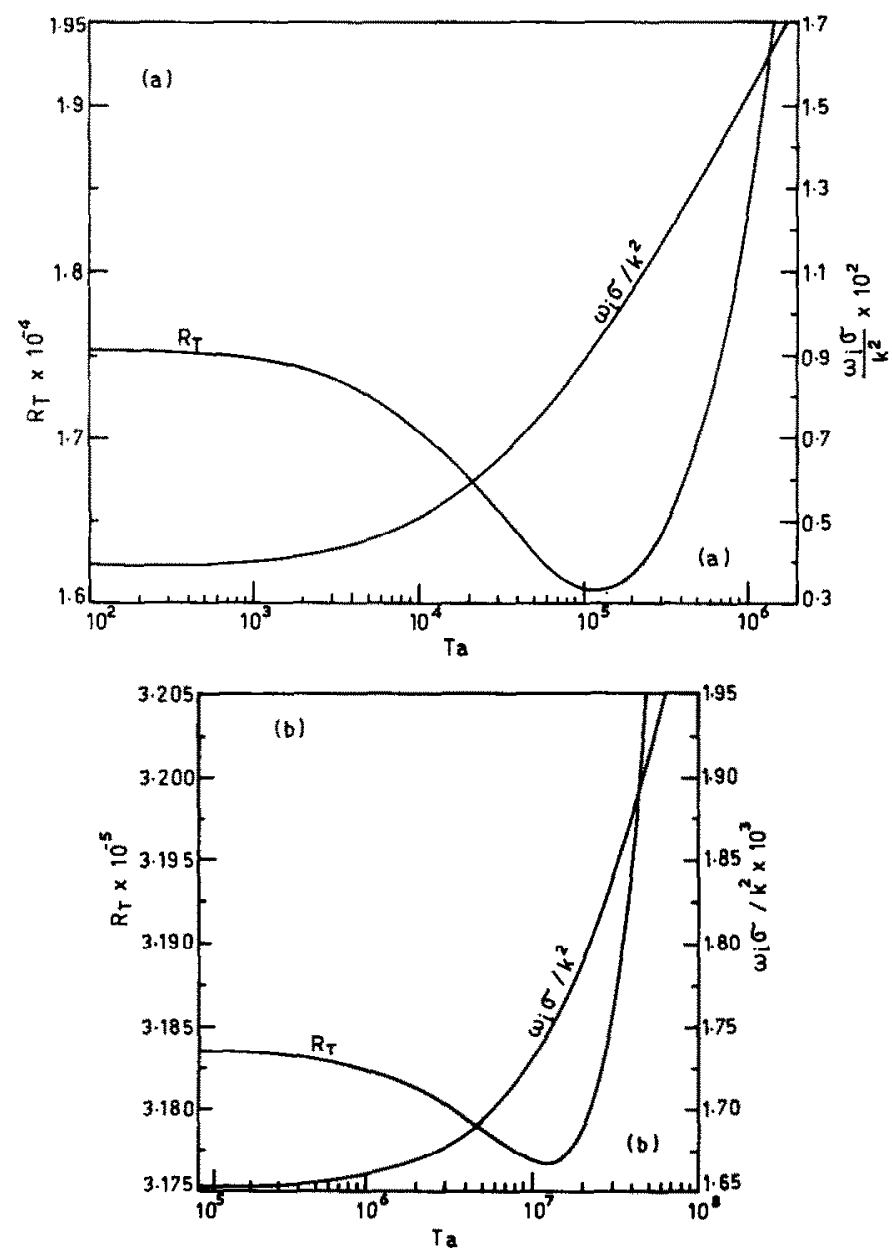

FiG. 3. Variation of $R_{\mathrm{T}}$ and $\omega_{\mathrm{i}} \sigma / k^{2}$ with $T a$. (a) $\tau=0.28, \sigma=0.025, R_{\mathrm{s}}=4500$ and $P_{1}=10^{-2}$; (b) $\tau=0.28$, $\sigma=0.002, R_{\mathrm{s}}=4 \times 10^{5}$ and $P_{\mathrm{i}}=10^{-3}$. 


\section{EXAMPLES OF STABILITY BOUNDARIES}

The work discussed in the previous section and that of Chakrabarti and Gupta [1], depicts only the effect of rotation and porous parameter on the onset of convection and is free from establishing its effect on stability boundaries. Therefore, in this section the stability boundaries are discussed by taking specific examples based on the values of $\tau, \sigma, P_{1}$ and $T a$ with the object of establishing the existence of salt-finger and diffusive regions.

We substitute $P=\mathrm{i} P_{i}$ into (13) and require $P_{i}$ to be real. This yields a hyperboloidal surface $\mathscr{H}$ in $\left(R_{\mathrm{T}}, R_{\mathrm{s}}\right.$, $R_{\mathrm{Ta}}$ ) space, whose analytical derivation is given in the Appendix. The plane boundary $\mathscr{P}$ and the surface $\mathscr{H}$ alone still do not determine fully the conditions under which marginal stable or oscillatory convection may occur, because there may be a possibility that the complex roots of the quartic characteristic equation (13) may become real roots without thcir parts passing through zero. Therefore, in this section the possible positions of the boundaries $\mathscr{P}$ and $\mathscr{H}$ are illustrated. We define the plane $\mathscr{S}$ given by

$$
R_{\mathrm{T}}-R_{\mathrm{s}}=0,
$$

on which the net density gradient is statically stable. By choosing $\tau=0.32, \sigma=0.025, P_{1}=10^{-2}, 10^{-3}$, $10^{-4}$ and $T a=10^{2}, 10^{3}, 10^{5}, 10^{6}$, the relevant portions of the intersections of $\mathscr{P}, \mathscr{H}$ and $\mathscr{F}$ are drawn in Figs. 4-6. The 'relevant portions' means those which describe a change in the mode of instability for the most unstable mode. In these figures the horizontally

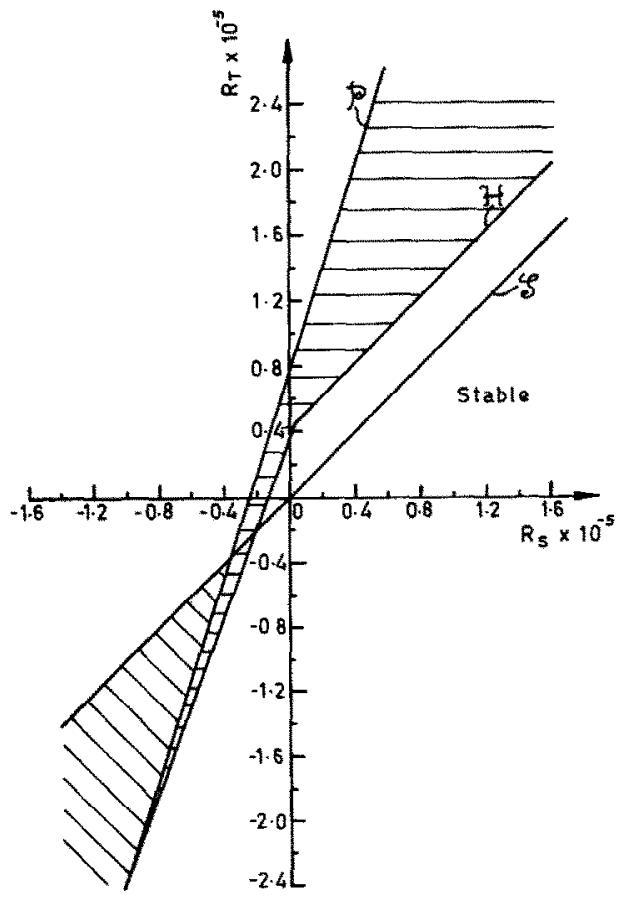

FIG. 4. Stability boundaries for $P_{1}=10^{-2}, \mathrm{Ta}=10^{6}, \tau=0.32$ and $\sigma=0.025$. The lines are explained in the text.

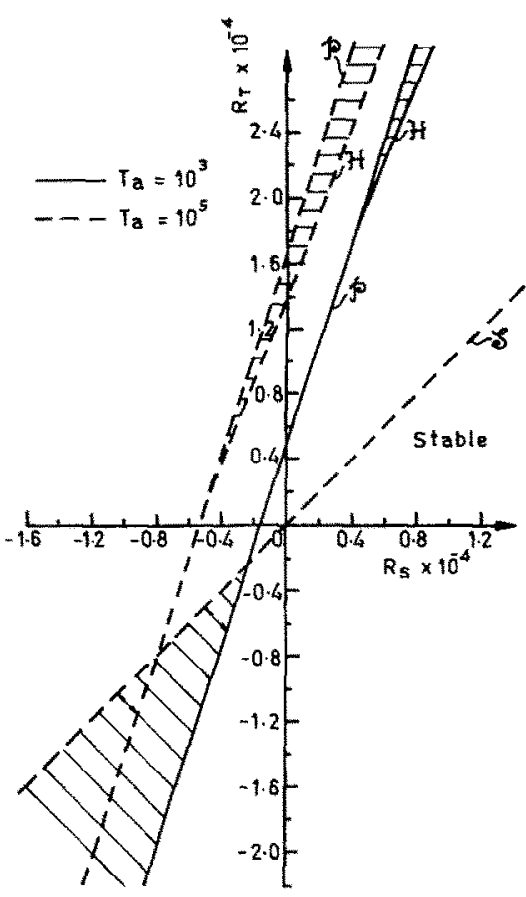

FIG. 5. Stability boundaries for two values of $T a$ for $P_{1}=10^{-2}, \tau=0.32$ and $\sigma=0.025$.

hatched regions give oscillatory modes and the oblique hatching shows conditions unstable to saltfingers. Comparing Figs. 4 and 5 we notice that as $T a$ increases, both salt-finger and overstable modes may be simultaneously unstable under a wide range of

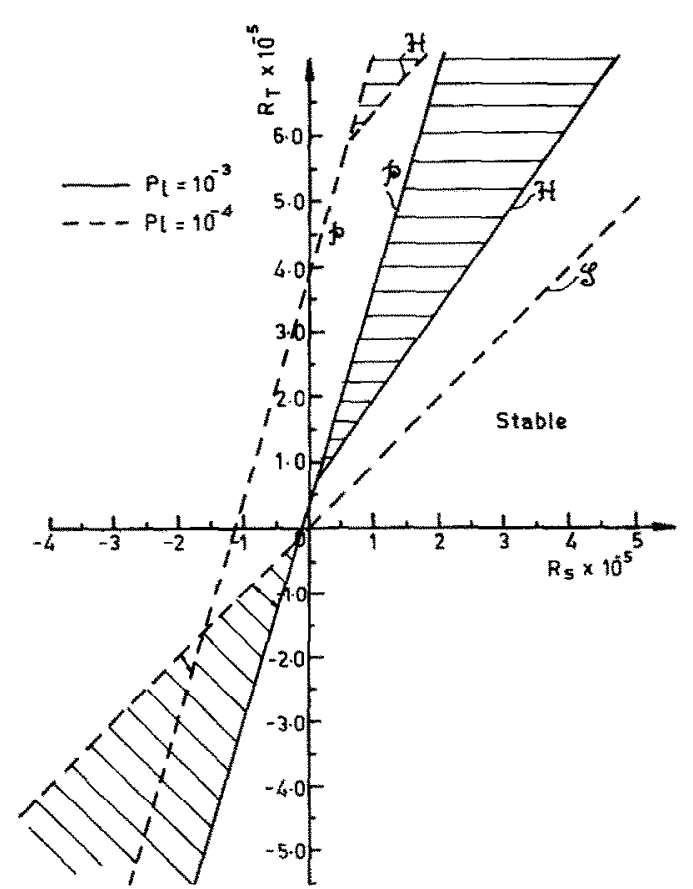

Fig. 6. Stability boundaries for two values of $P_{1}$ for $T a=10^{2}$, $\tau=0.32$ and $\sigma=0.025$. 
conditions for $P_{1}=10^{-2}$. The effect of rotation and porous parameter on stability boundaries is depicted in Figs. 5 and 6, respectively. We found that as $\mathrm{Ta}$ increases it stabilizes the salt-finger region and also increases the region of stability, i.e. rotation makes the system more stable. Figure 6 depicts that as $P_{1}$ decreases the region of stability increases. Also decrease in $P_{1}$ stabilizes the region of salt-finger.

\section{PARAMETRIC PERTURBATION METHOD}

In the previous sections we have considered only the isothermal boundaries. In this section we consider the behaviour of the critical Rayleigh number with respect to $R_{\mathrm{s}}, P_{1}$ boundary parameter (i.e. Biot number) and $T a^{1 / 2}$. As an extra result we also obtain expressions for obtaining first order effects on the eigenfunctions. We employ a method which has been used by Narayanan [21] and Shivakumara et al. [15]. These first-order effects can be calculated using a modified Greens matrix as the kernel of a matrix differential operator (see [22]).

From the linearized version of $(7)$ to $(10)$ we define an operator $L$ as follows : condition to (24) we get

$$
\frac{\pi \alpha}{\sigma}\left(\frac{\hat{R}_{\mathrm{T}}^{\mathrm{c}}}{R_{\mathrm{T}}^{\mathrm{c}}}-\frac{\tau}{R_{\mathrm{s}}}\right) \int_{V}|\psi|^{2} \mathrm{~d} v=0 .
$$

This yields the condition that $R_{\mathrm{T}}^{\mathrm{c}}$ increases if $R_{\mathrm{s}}$ increases. This means that a stabilizing concentration gradient causes an increase in the critical Rayleigh number for thermal motions. Equation (25) can be used to solve for $\hat{\mathbf{Q}}$ and we thus obtain the first-order effect of an increase in $R_{\mathrm{s}}$ about some known value.

The dependence on boundary parameters will help us in determining the behaviour of thermal Rayleigh number with Biot $(B i)$ and Sherwood $(S h)$ numbers. We show this because an earlier analysis is for the special case of Biot and Sherwood numbers going to infinity. We shall easily see that the obtained Rayleigh numbers are upper bounds for finite Biot and Sherwood numbers.

We may differentiate (24) and the attendant boundary conditions with respect to Biot number. This yields

$$
L \overline{\mathbf{Q}}=\left[0,0, \frac{\pi \alpha}{\sigma} \psi \frac{\bar{R}_{\mathrm{T}}^{\mathrm{c}}}{R_{\mathrm{T}}^{\mathrm{c}}}, 0\right]
$$

$$
L \equiv\left[\begin{array}{cccc}
D^{4}-\left(2 \pi^{2} \alpha^{2}+P_{1}^{-1}\right) D^{2} & T a^{1 / 2} D & -\pi \alpha \sigma^{-1} & \pi \alpha \sigma^{-1} \tau \\
+\pi^{2} \alpha^{2}\left(\pi^{2} \alpha^{2}+P_{1}^{-1}\right) & \left(D^{2}-\pi^{2} \alpha^{2}-P_{1}^{-1}\right) & 0 & 0 \\
-T a^{1 / 2} D & 0 & \frac{\left(D^{2}-\pi^{2} \alpha^{2}\right)}{R_{\mathrm{T}} \sigma^{2}} & 0 \\
-\pi \alpha \sigma^{-1} & 0 & 0 & \frac{-\tau^{3}\left(D^{2}-\pi^{2} \alpha^{2}\right)}{R_{\mathrm{s}} \sigma^{2}}
\end{array}\right]
$$

where $D=\partial / \partial z$.

Now, if we define $\mathbf{Q}^{\mathfrak{t}} \equiv[\psi, V, \theta, \Sigma]$, where the four vector represents the dependent variables then from (7) - (10) we have

$$
L \mathbf{Q}=\mathbf{0} \text {. }
$$

To visualize the variation of $R_{\mathrm{T}}^{\mathrm{c}}$ with respect to $R_{\mathrm{s}}$ we differentiate (24) with respect to $R_{\mathrm{s}}$ and obtain

$$
L \hat{\mathbf{Q}}=\hat{\mathbf{h}}
$$

where

$$
\hat{\mathbf{h}}^{\mathrm{t}}=\left[0,0, \frac{\pi \alpha}{\sigma} \psi \frac{\hat{R}_{\mathrm{T}}^{\mathrm{c}}}{R_{\mathrm{T}}^{\mathrm{c}}}, \quad-\frac{\pi \alpha}{\sigma} \psi \tau / R_{\mathrm{s}}\right]
$$

Now, if

$$
\langle\mathbf{a}, \mathbf{b}\rangle \equiv \int_{V} a^{* t} \cdot b \mathrm{~d} v
$$

is a suitable inner product where the asterisks represent complex conjugates then we can show that $L$ is self-adjoint and the boundary conditions are also self-adjoint. Thus applying a Fredholm alternative where ( ${ }^{-}$) represents derivatives with respect to $B i$ and at the boundary we have

$$
D \bar{\theta}+B i \bar{\theta}+\theta=0 .
$$

Thus on applying the solvability condition to (28) in the light of (29) we get after much algebraic manipulation

$$
\frac{\pi \alpha}{\sigma} \frac{\bar{R}_{\mathrm{T}}^{\mathrm{c}}}{\boldsymbol{R}_{\mathrm{T}}^{\mathrm{c}}} \int_{V} \theta^{*} \psi \mathrm{d} v=\frac{1}{R_{\mathrm{T}}^{\mathrm{c}} \sigma^{2}} \int_{V}-|\theta|^{2} \mathrm{~d} v .
$$

On using the energy equation we will get from (30)

$$
\frac{\partial l_{\mathrm{n}} R_{\mathrm{T}}^{\mathrm{c}}}{\partial B i}>0 .
$$

Thus if $R_{\mathrm{T}}^{\mathrm{c}}<0$ (such as the case of heated from above) then $R_{\mathrm{T}}^{\mathrm{c}}$ will decrease with increase in $B i$. In most cases we are concerned with the case of fluid heated from below and so the case of $B i$ tending to $\infty$ provides an upper bound on $R_{\mathrm{T}}^{\mathrm{c}}$. Equation (28) is a vehicle for calculating the first-order effects and a modified Greens matrix is needed for these calculations (see [22]). 
We finally consider the effect of $T a$ and $P_{\mathrm{I}}^{-1}$ on $R_{\mathrm{T}}^{\mathrm{c}}$ and some interesting conclusions will be drawn. On differentiating (24) with respect to $T a^{1 / 2}$ we get

$$
L \hat{\mathbf{Q}}=\hat{\mathbf{h}}
$$

where

$$
\hat{\mathbf{h}}^{\mathrm{t}}=\left[-D V, D \psi, \frac{\pi \alpha}{\sigma} \psi \frac{\hat{R}_{\mathrm{T}}^{\mathrm{c}}}{R_{\mathrm{T}}^{\mathrm{c}}}, 0\right]
$$

and represents derivatives with respect to $\mathrm{Ta}^{1 / 2}$. On applying the solvability conditions we get

$$
\begin{aligned}
& T a^{1 / 2} \frac{\pi \alpha}{\sigma} \frac{\hat{R}_{\mathrm{T}}^{\mathrm{c}}}{R_{\mathrm{T}}^{\mathrm{c}}} \int_{V} \psi \theta^{*} \mathrm{~d} v \\
& =-\int_{V}\left|D^{2} \psi\right|^{2} \mathrm{~d} v+\frac{\pi \alpha}{\sigma} \int_{V} \psi^{*} \theta \mathrm{d} v \\
& -\left(2 \pi^{2} \alpha^{2}+P_{1}^{-1}\right) \int_{V}|D \psi|^{2} \mathrm{~d} v \\
& -\pi^{2} \alpha^{2}\left(\pi^{2} \alpha^{2}+P_{1}^{-1}\right) \int_{V}|\psi|^{2} \mathrm{~d} v \\
& -\frac{\tau \pi \alpha}{\sigma} \int_{V} \psi^{*} \Sigma \mathrm{d} v-T a^{1 / 2} \int_{V} V^{*} D \psi \mathrm{d} v
\end{aligned}
$$

We use the energy and species continuity equation to arrive at the conclusion that $\hat{R}_{\mathrm{T}}^{\mathrm{c}}>0$ if $R_{\mathrm{s}}$ and $R_{\mathrm{T}}^{\mathrm{c}}$ are negative and positive, respectively. That is we can only say that an increase in rotation causes an increase in $R_{\mathrm{T}}^{\mathrm{c}}$ if we have the most unstable case.

On differentiating (24) with respect to $P_{1}^{-1}$, we get

$$
L \tilde{\mathbf{Q}}=\tilde{\mathbf{h}},
$$

where

$$
\tilde{\mathbf{h}}^{\mathrm{t}}=\left[\left(D^{2}-\pi^{2} \alpha^{2}\right) \psi, V, \frac{\pi \alpha \psi}{\sigma} \frac{\tilde{R}_{\mathrm{T}}^{\mathrm{c}}}{R_{\mathrm{T}}^{\mathrm{c}}}, 0\right]
$$

and $\sim$ represents derivatives with respect to $P_{1}^{-1}$. On applying the solvability condition we get

$$
\begin{aligned}
\frac{\pi \alpha}{\sigma} \frac{\tilde{R}_{\mathrm{T}}^{\mathrm{c}}}{R_{\mathrm{T}}^{\mathrm{c}}} \int_{V} \psi \theta^{*} \mathrm{~d} v= & \int_{V}|D \psi|^{2} \mathrm{~d} v \\
& +\pi^{2} \alpha^{2} \int_{V}|\psi|^{2} \mathrm{~d} v-\int_{V}|v|^{2} \mathrm{~d} v .
\end{aligned}
$$

This yields the condition that $R_{\mathrm{T}}^{\mathrm{c}}$ increases if $P_{\mathrm{I}}$ decreases. This means that critical Rayleigh number increases as the porous medium becomes more dense, because the resistance offered to the flow per unit volume of the medium is quite large.

\section{FINITE-AMPLITUDE CONVECTION WITH A LIMITED REPRESENTATION}

Linear theory discussed in the previous sections predicts only the criterion for the onset of convection. But for flows with $R_{\mathrm{T}}>R_{\mathrm{T}}^{\mathrm{c}}$, the linear stability analy- sis is not valid and we have to take into account the non-linear effects. In this section, we discuss the nonlinear stability of convection of a two-component, fluid-saturated porous layer in the presence of rotation using a severely truncated representation of Fourier series (see [19]).

It is important to note that the effect of nonlinearity is to distort the zonal velocity, temperature and concentration fields through the interaction of $\psi$ and $V, \psi$ and $\theta$ and $\psi$ and $\Sigma$, respectively. As a result, a component of the form $\sin (2 \pi \alpha x)$ in the first case and of the form $\sin (2 \pi z)$ in the latter two cases will be generated.

Therefore, the minimal system which describes finitc-amplitude convection is of the form

$$
\begin{gathered}
\psi=A(t) \sin \pi \alpha x \sin \pi z \\
\theta=B(t) \cos \pi \alpha x \sin \pi z+C(t) \sin 2 \pi z \\
\Sigma=D(t) \cos \pi \alpha x \sin \pi z+E(t) \sin 2 \pi z \\
V=F(t) \sin \pi \alpha x \cos \pi z+G(t) \sin 2 \pi \alpha x
\end{gathered}
$$

where the amplitudes $A, B, C, D, E, F$ and $G$ are generally functions of time and are to be determined by the dynamics of the system. Substituting (37)-(40) into (7)-(10) and equating the coefficients of like terms we get the following set of time-dependent, non-linear ordinary differential equations :

$$
\begin{gathered}
\frac{\mathrm{d} A}{\mathrm{~d} t}=-\sigma k^{2} \eta A+\frac{\pi \sigma T a^{1 / 2}}{k^{2}} F+\frac{\pi \alpha \tau}{k^{2}} D-\frac{\pi \alpha}{k^{2}} B \\
\frac{\mathrm{d} B}{\mathrm{~d} t}=-k^{2} B-\pi \alpha \sigma R_{\mathrm{T}} A-\pi^{2} \alpha \sigma A C \\
\frac{\mathrm{d} C}{\mathrm{~d} t}=-4 \pi^{2} C+\frac{\pi^{2} \alpha \sigma}{2} A B \\
\frac{\mathrm{d} D}{\mathrm{~d} t}=-\tau k^{2} D-\pi^{2} \alpha \sigma A E-\frac{\sigma R_{\mathrm{s}}}{\tau} \pi \alpha A \\
\frac{\mathrm{d} E}{\mathrm{~d} t}=-4 \pi^{2} \tau E+\frac{\pi^{2} \alpha \sigma}{2} A D \\
\frac{\mathrm{d} F}{\mathrm{~d} t}=-k^{2} \sigma \eta F+\pi^{2} \alpha \sigma A G-\pi \sigma T a^{1 / 2} A \\
\frac{\mathrm{d} G}{\mathrm{~d} t}=-\left(4 \pi^{2} \alpha^{2}+1 / P_{1}\right) \sigma G-\frac{\pi^{2} \alpha \sigma}{2} A F .
\end{gathered}
$$

This seventh-order Lorenz model of double-diffusive convection in a porous medium in the presence of rotation provides a good example whose solutions are at any rate qualitatively similar to those of the partial differential equations from which they are derived (see [23, 24]). Also this truncated system is useful in establishing the details of the bifurcation structure and, in particular, of the transition from oscillatory to steady motions.

\subsection{Steady, finite-amplitide convection}

The above set of non-linear ordinary differential equations for the general, time-dependent variables is 
not amenable to analytical treatment and we have to solve it numerically. However, we note that, for the steady case $(\partial / \partial t=0)$ these equations reduce to a set of algebraic equations and eliminating all amplitudes (except for $A$ ) yields, after some algebraic simplification

$$
A\left[\left(\frac{A^{2}}{8}\right)^{3}+a_{2}\left(\frac{A^{2}}{8}\right)^{2}+a_{1}\left(\frac{A^{2}}{8}\right)+a_{0}\right]=0
$$

where

$$
\begin{aligned}
a_{0}= & \frac{\tau^{2}}{\beta \pi^{4} \sigma^{4} \alpha^{2}}\left[\frac{\pi^{4}\left(\alpha^{2}+1\right)^{3}}{\alpha^{2}} \eta+\frac{R_{\mathrm{ra}}}{\sigma \eta \alpha^{2}}-R_{\mathrm{T}}+\frac{R_{\mathrm{s}}}{\tau}\right] \\
a_{1}= & \frac{\left(\alpha^{2}+1\right)}{k^{4} \eta \beta \sigma^{4} \alpha^{2}}\left[k^{4}\left(\alpha^{2}+1\right) \eta^{2}\left(\sigma^{2}\left(1+\tau^{2}\right)+\frac{\beta \tau^{2}}{\eta \alpha^{2}}\right)\right. \\
& +\sigma R_{\mathrm{Ta}}\left(1+\tau^{2}\right)+\sigma^{2} \alpha^{2}\left(-R_{\mathrm{T}}+R_{\mathrm{s}} \tau\right)+\beta \tau^{2} \\
& \left.\times\left(-R_{\mathrm{T}}+\frac{R_{\mathrm{s}}}{\tau}\right)\right] \\
a_{2}= & \frac{1}{k^{4} \eta \beta \sigma^{2} \alpha^{2}}\left[k^{4}\left(\alpha^{2}+1\right) \eta\left(\sigma^{2} \alpha^{2} \eta+\beta \tau^{2}+\beta\right)\right. \\
& \left.+\sigma R_{\mathrm{Ta}} \alpha^{2}+\beta \alpha^{2}\left(-R_{\mathrm{T}}+R_{\mathrm{s}} \tau\right)\right] .
\end{aligned}
$$

and

$$
\beta=\frac{\pi^{2} \alpha^{2}}{\pi^{2} \alpha^{2}+4 P^{-1}}
$$

The solution $A=0$, corresponds to pure conduction, which we know to be a possible solution though it is unstable when $R_{\mathrm{T}}$ is sufficiently large. The remaining solutions are determined by solving the cubic polynomial in $A^{2} / 8$ numerically. So we know the amplitude and hence we can find the corresponding heat and mass transfer.

\subsection{Convective heat and mass transport}

In the study of double-diffusive convection in a porous medium in the presence of Coriolis forces, the determination of heat and mass transport across the layer plays a very important role in many practical problems discussed in Section 1 and also it paves the way to understand the more complicated problems. We calculate the heat and mass transport in terms of Nusselt number, $N u$, and the solute Nusselt number, $N u^{s}$, as functions of $R_{\mathrm{T}}, R_{s}, T a, P_{1}, \sigma$ and $\tau$. The Nusselt number is defined as the ratio of the total vertical heat flux, $H$, to the conductive, vertical heat flux. In the steady state, the vertical heat flux is independent of the vertical coordinate, $z$, and can be evaluated as

$$
H=-\kappa\langle\partial \tilde{T} / \partial Z\rangle_{t=0},
$$

where the angular bracket corresponds to a horizontal average. With the definition of $\tilde{T}$ from $(1),(49)$ can be written as

$$
N u=\frac{H d}{\kappa \Delta T}=1-\frac{1}{R_{\mathrm{T}}} 2 \pi C .
$$

Similarly, the solute Nusselt number $N u^{s}$ is defined by

$$
N u^{\mathrm{s}}=1-\frac{1}{\left(R_{\mathrm{s}} / \tau\right)} 2 \pi E
$$

where $B, C, D, E$ are given by (41)-(47). If the heat and mass transport are purely by conduction for which $A=0$ and $B, C, D, E, F$ and $G$ are all zero, then equations (50) and (51) show that $N u$ and $N u^{s}$ are unity.

Our purpose in this section is to determine the quantitative effect of rotation, salinity gradient, diffusivity ratio, porous parameter, and Prandtl number on heat and mass transport by thermal and mass advection terms for which $A \neq 0$. The results are shown in Table 2 and also depicted in Figs. 7-11. In Table 2 the values of $N u$ and $N u^{5}$ for several values of $R_{\mathrm{T}}$ for $T a=10^{6}$, $P_{1}=10^{-2}, R_{\mathrm{s}}=10^{4}, \tau=0.32$ and $\sigma=0.2,1.0$ and 10.0 are tabulated. From this table we can see that $N u$ and $N u^{s}$ increase with an increase in $R_{\mathrm{T}}$ and decrease in $\sigma$. From this table we also note that the values of $N u$ and $N u^{s}$ for $\sigma=1$ and $\sigma=10$ are close to each other than that of $\sigma=0.2$. Although the presence of a stabilizing gradient of solute will serve to inhibit the onset of convection, the strong finite-amplitude motions which exist for moderate Rayleigh numbers tend to mix the solute and distribute it so that the
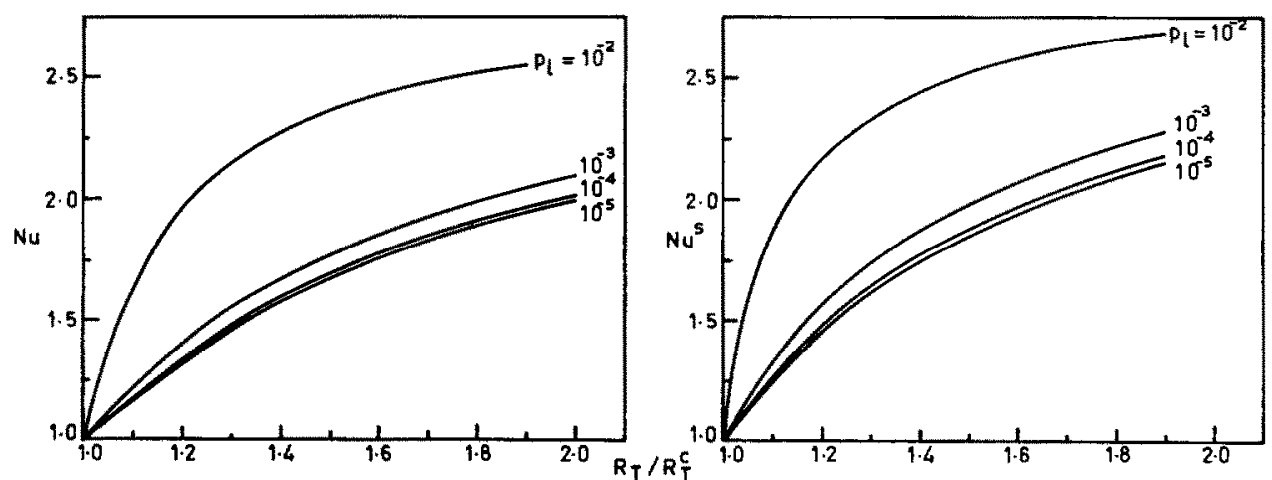

FIG. 7. Variation of $N u$ and $N u^{4}$ with porous parameter for $\tau=0.81, \sigma=0.2, T a=10^{6}$ and $R_{\mathrm{s}}=10^{4}$. 
Table 2. $N u$ and $N u^{s}$ (the upper value in each pair) for $T a=10^{6}, P_{1}=10^{-2}$ and $\tau=0.32$ with different values of $\sigma$

\begin{tabular}{ccccc}
\hline & \multicolumn{4}{c}{$R_{\mathrm{s}}=10^{4}$} \\
\cline { 2 - 4 }$R_{\mathrm{T}}$ & $\sigma=0.2$ & $\sigma=1.0$ & $\sigma=10.0$ & $\begin{array}{c}R_{\mathrm{s}}=0 \\
\sigma=0.2\end{array}$ \\
\hline \multirow{2}{*}{$7.5 \times 10^{4}$} & 1.0000 & 1.0000 & 1.0000 & \\
& 1.0000 & 1.0000 & 1.0000 & 1.0006 \\
$8 \times 10^{4}$ & 1.0000 & 1.0000 & 1.0000 & \\
& 1.0000 & 1.0000 & 1.0000 & 1.2599 \\
$8.5 \times 10^{4}$ & 1.0000 & 1.0000 & 1.0000 & \\
& 1.0000 & 1.0000 & 1.0000 & 1.5522 \\
$9.0 \times 10^{4}$ & 1.0000 & 1.0000 & 1.0000 & \\
& 1.0000 & 1.0000 & 1.0000 & 1.7559 \\
$9.5 \times 10^{4}$ & 2.6342 & 1.0000 & 1.0000 & \\
& 1.6277 & 1.0000 & 1.0000 & 1.9075 \\
$1.0 \times 10^{5}$ & 2.7634 & 1.0000 & 1.0000 & \\
& 1.8657 & 1.0000 & 1.0000 & 2.0251 \\
$1.2 \times 10^{5}$ & 2.8874 & 2.6449 & 2.6304 & \\
& 2.2637 & 1.6435 & 1.6224 & 2.3159 \\
$1.4 \times 10^{5}$ & 2.9243 & 2.7668 & 2.7554 & \\
& 2.4450 & 1.8737 & 1.8472 & 2.4719 \\
$1.6 \times 10^{5}$ & 2.9428 & 2.8253 & 2.8152 & \\
& 2.5532 & 2.0336 & 2.0030 & 2.5697 \\
$2.0 \times 10^{5}$ & 2.9614 & 2.8838 & 2.8751 & \\
& 2.6776 & 2.2479 & 2.2119 & 2.6857 \\
$2.4 \times 10^{5}$ & 2.9708 & 2.9134 & 2.9055 & \\
& 2.7476 & 2.3868 & 2.3473 & 2.7524 \\
$3.0 \times 10^{5}$ & 2.9787 & 2.9379 & 2.9306 & \\
& 2.8094 & 2.5233 & 2.4806 & 2.8121 \\
\hline
\end{tabular}

interior layers of the fluid are more neutrally stratified. Therefore, as $R_{\mathrm{T}}$ becomes large the values of $\mathrm{Nu}$ are seen to approach those with no stabilizing gradient $\left(R_{s}=0\right)$.

It is further found that subcritical motions are possible for certain values of $T a, P_{1}, \sigma, \tau$ and $R_{\mathrm{s}}$. The resulting delay of the onset of instability by infinitesimal disturbances is the main reason for the subcritical finite-amplitude instability. For example, for $R_{s}=10^{4}, \tau=0.81, P_{1}=10^{-2}$ and $\sigma=0.2$ subcritical motions are possible up to $T a<10^{6}$ and also it is found that no subcritical motions are possible, in general, for small values of porous parameters. However, subcritical motions are possible even for small values of $P_{1}$ only when $\tau$ is small. For example, when $\tau=0.01$, subcritical motions are possible even for $P_{1}=10^{-4}$, $10^{-5}$. In Figs. 7 and 8 we exhibit the values of $\mathrm{Nu}$ and

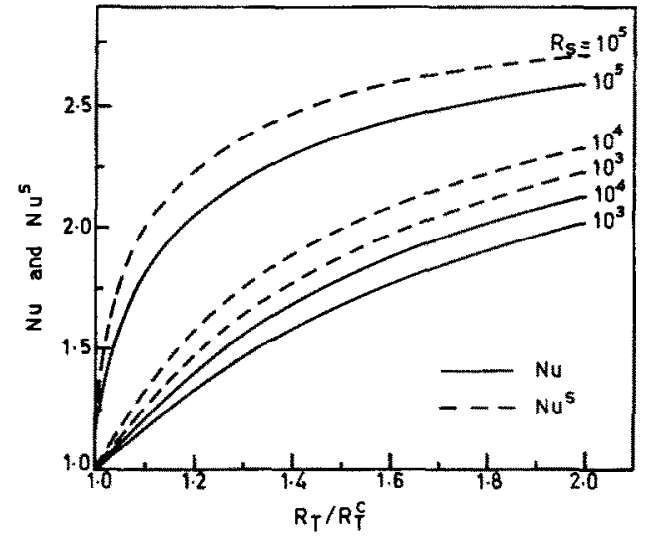

Fig. 9. Variation of $N u$ and $N u^{s}$ with $R_{\mathrm{s}}$ for $\tau=0.81, \sigma=0.2$, $T a=10^{6}$ and $P_{1}=10^{-3}$.

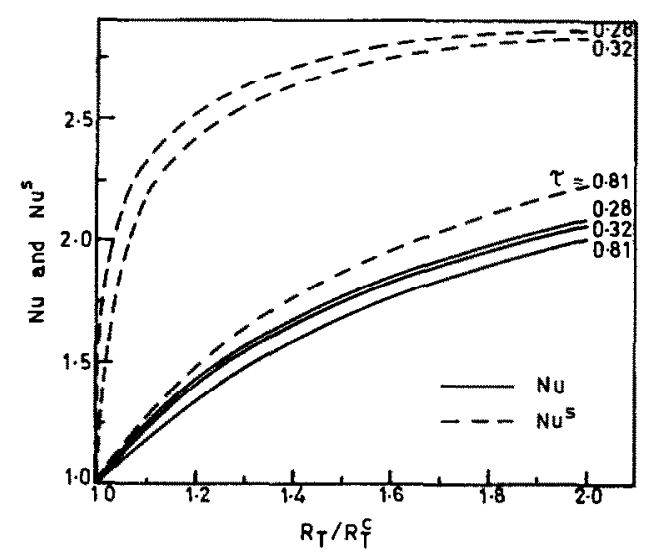

Fig. 10, Variation of $\mathrm{Nu}$ and $\mathrm{Nu}^{\mathrm{s}}$ with $\tau$ for $\sigma=0.2, T a=10^{4}$, $P_{1}=10^{-4}$ and $R_{\mathrm{s}}=10^{4}$.

$N u^{s}$ vs $R_{\mathrm{T}} / R_{\mathrm{T}}^{c}$ in the form of a graph for different values of $P_{1}$ and $T a$, respectively. Figure 7 reveals that at any given value of $R_{\mathrm{T}} / R_{\mathrm{T}}^{\mathrm{c}}$ the Nusselt number decreases with decrease in $P_{1}$, because the resistance offered to the flow by the element in unit volume of the fluid is sufficiently large so that the system becomes more stable. From Fig. 8, however, it is clear that the Nusselt number decreases with increasing $T a$. Thus, in the case of steady motion the effect of rotation is
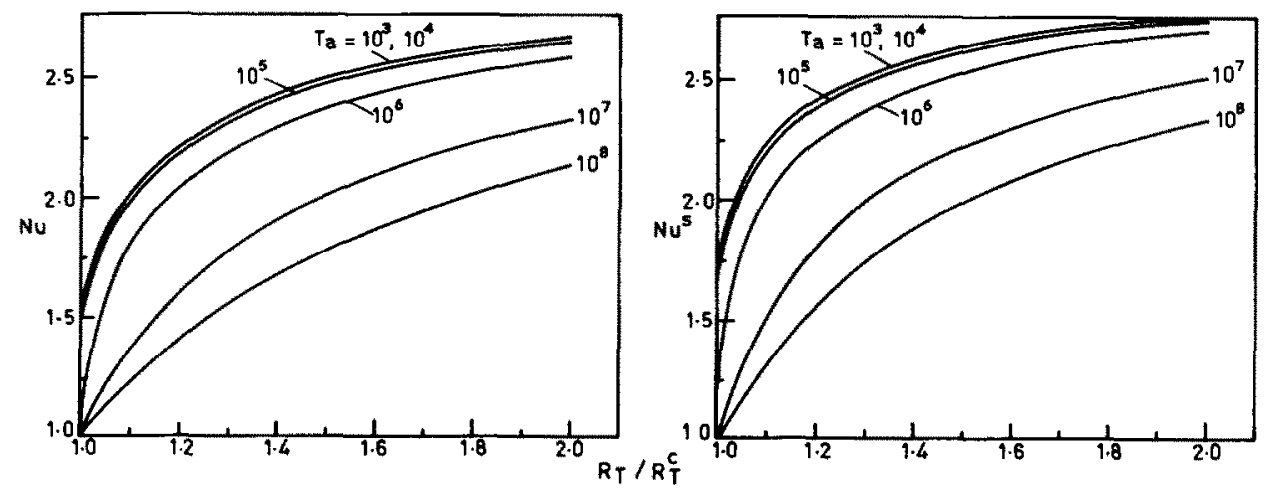

Fig. 8. Variation of $N u$ and $N u^{s}$ with Taylor number for $\tau=0.81, \sigma=0.2, P_{1}=10^{-3}$ and $R_{\mathrm{s}}=10^{5}$. 

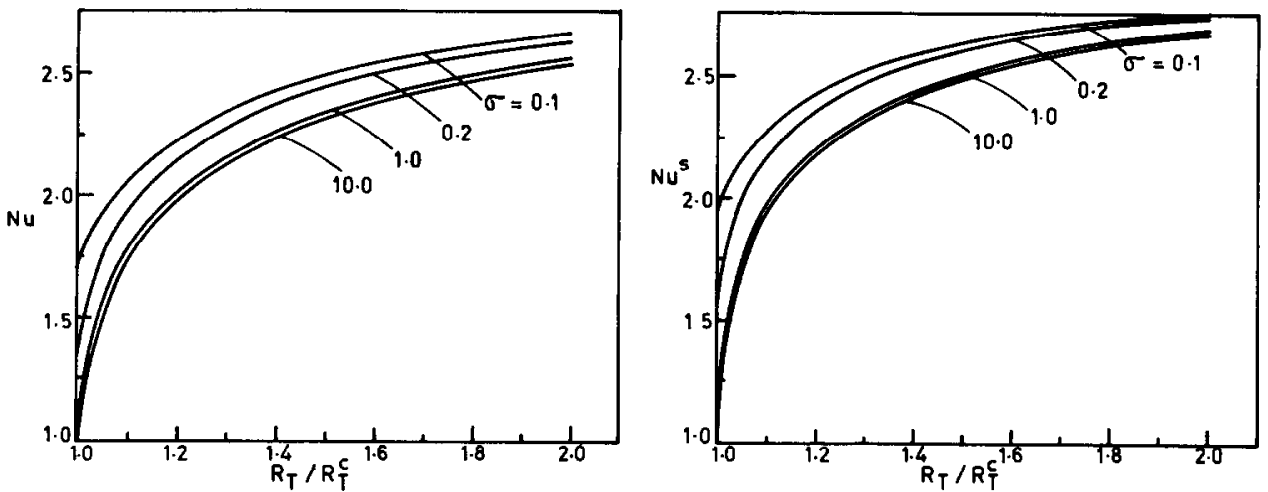

FIG. 11. Variation of $N u$ and $N u^{\mathrm{s}}$ with $\sigma$ for $\tau=0.81, \mathrm{Ta}=10^{4}, P_{1}=10^{-2}$ and $R_{\mathrm{s}}=10^{4}$.

to make the system more stable in addition to the stabilizing effect of porous parameter. From this figure it is also clear that for $T a=10^{3}$ and $10^{4}$, the heat or mass transport are almost the same. This may be due to the dominating effect of the other parameters like $P_{\mathrm{l}}, R_{\mathrm{s}}$ and $\tau$. Figure 9 is the plot of $N u$ and $N u^{\mathrm{s}}$ for different values of $R_{\mathrm{s}}$ for $T a=10^{6}, \sigma=0.2$, $\tau=0.81$ and $P_{1}=10^{-3}$. From this figure we see that the effect of an increase in $R_{\mathrm{s}}$ is to increase heat and mass transport.

Figures 10 and 11 clearly depict the effect of $\tau$ and $\sigma$ on heat and mass transport. From Fig. 10, it is clear that heat and mass transport increases with decrease in $\tau$. From this figure it is also evident that the dependence of $\mathrm{Nu}$ on diffusivity ratio is weak, at least for the range shown. However, the situation is different in the case of $N u^{s}$. Figure 11 clearly depicts the effect of $\sigma$ on heat and mass transport. The values of $N u$ and $N u^{s}$ for $\sigma=1$ and $\sigma=10$ are closer to each other than those for $\sigma=0.2$ and 0.1 . Also it is found that as $P_{1}$ decreases the effect of $\sigma$ on heat and mass transfer is not so significant.
6.3. Streamlines, isotherms, isohalines and zonal velocity distribution

Streamlines drawn for different values of $P_{1}$ and $T a$ are shown in Fig. 12. From this figure we can see that as $P_{1}$ and $T a$ decrease the wavelength of the cell, $1 / \alpha$, decreases, i.e. cells will contract.

The isotherm and isohaline patterns are shown in Figs. 13 and 14 for three values of $P_{1}$ for $T a=10^{4}$, $\sigma=0.2, \tau=0.81$ and $R_{\mathrm{s}}=10^{4}$. From these figures it is clear that isotherms and isohalines in the centre of the cell are more or less vertical (i.e. anvil-shaped) and become horizontal near $x=0$ and $x=1 / \alpha$ for $P_{1}=10^{-2}$, i.e. cold fluid sinks and spreads out close to the bottom boundary and plumes of warm fluid spread close to the top boundary. But for $P_{1}=10^{-3}$ and $10^{-4}$ the anvil-shaped plumes are inhibited as can be seen in Figs. 13(b), (c) and 14(b), (c). This is because of the strong constraining effect of the porous parameter which suppresses the convection and hence inhibits the formation of anvil-shaped plumes.

In Figs. 15(a), (b) we show a pattern of contour lines for the zonal velocity, $V$, for the flow corresponding to
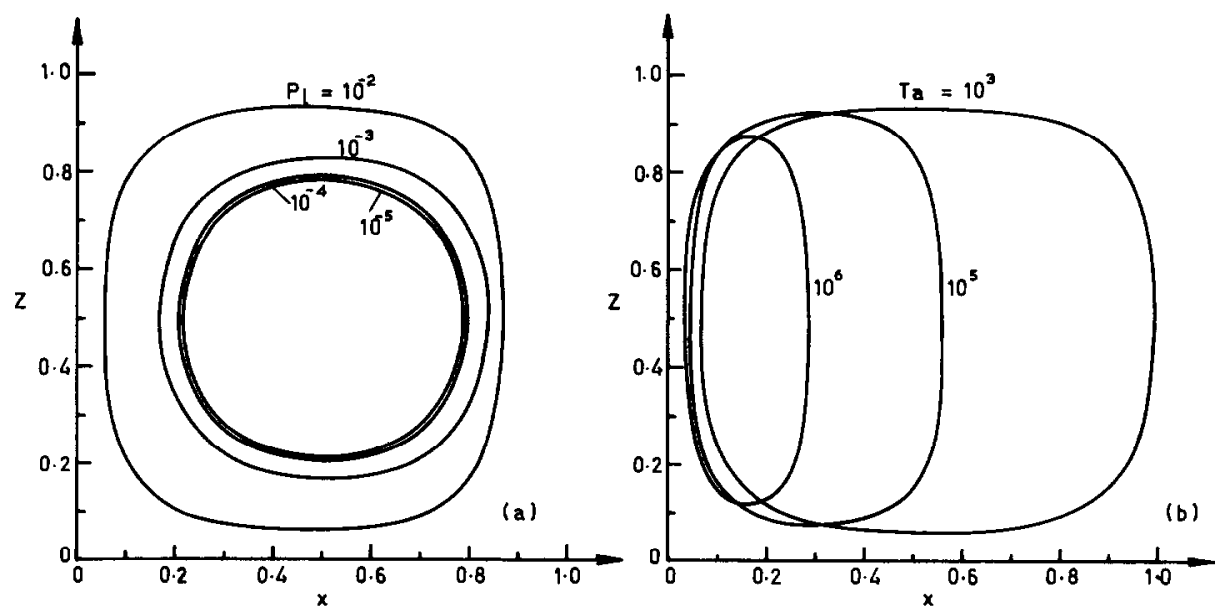

Fig. 12. Streamlines for $\psi=4$ and $R_{\mathrm{T}}=1.1 R_{\mathrm{T}}^{\mathrm{c}}$ (a) with different values of porous parameter for $\tau=0.81$ $\sigma=0.2, T a=10^{4}$, and $R_{s}=10^{4}$; (b) with different values of Taylor number for $\tau=0.81, \sigma=0.2, P_{1}=10^{-2}$ and $R_{\mathrm{s}}=10^{4}$. 

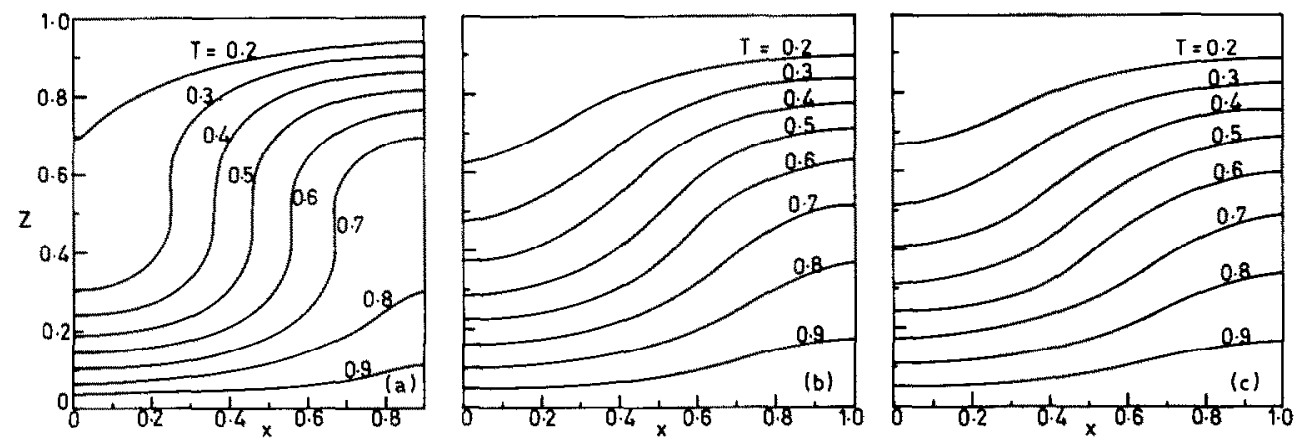

FIG. 13. Isotherms for $\tau=0.81, \sigma=0.2, T a=10^{4}, R_{\mathrm{s}}=10^{4}$ and $R_{\mathrm{T}}=1.1 R_{\mathrm{T}}^{\mathrm{c}}$ : (a) $P_{1}=10^{-2}$, (b) $P_{1}=10^{-3}$ and (c) $P_{1}=10^{-4}$.

the isotherm patterns of Figs. 13(a), (b), respectively. From Fig. 15(a) it is clear that there is a strong shift of maximum and minimum velocities toward the lateral boundaries. For $P_{1}=10^{-3}$, however, the flow shows a nearly horizontal symmetry. This feature may be due to the stronger constraint imposed by the porous parameter, with the horizontal temperature gradient more nearly balanced by the vertical shear of the zonal velocity.

\subsection{Unsteady, finite-amplitude convection}

In this section the behaviour of non-linear, periodic solutions are investigated with the object of understanding the transition from periodic oscillations to behaviour that is apparently chaotic (i.e. solutions are aperiodic and depend sensitively on the initial conditions), and to predict the amount of heat and mass transfer. For this purpose, we integrate (41)(47) numerically using the Runge-Kutta-Gill method satisfying the following initial conditions :

$$
\begin{gathered}
A=0.1, \quad B=1, \quad C=0, \quad D=1, \\
E=0, \quad F=1 \quad \text { and } \quad G=0 .
\end{gathered}
$$

The system (41)-(47) possesses an important symmetry, for it is invariant under the transformation

$(A, B, C, D, E, F, G)$

$$
\rightarrow(-A,-B, C,-D, E,-F, G) .
$$

Moreover, these equations describe a contraction mapping principle in the seven-dimensional space, since

$$
\begin{aligned}
\frac{\partial \dot{A}}{\partial A} & +\frac{\partial \dot{B}}{\partial B}+\frac{\partial \dot{C}}{\partial C}+\frac{\partial \dot{D}}{\partial D}+\frac{\partial \dot{E}}{\partial E}+\frac{\partial \dot{F}}{\partial \boldsymbol{F}} \\
& +\frac{\partial \dot{G}}{\partial G}=-\left[k^{2}\{2 \sigma \eta+1+\tau\}\right. \\
& \left.+4 \pi^{2}(1+\tau)+\left(4 \pi^{2} \alpha^{2}+1 / P_{1}\right) \sigma\right]<0
\end{aligned}
$$

where the dot denotes the derivative with respect to time. This implies that trajectories may be attracted to fixed points, to limit cycles or, possibly, to a strange attractor.

The results of the numerical study have been documented in Table 3, considering, for the sake of comparison, a representative set of values from the Table 2 . It is clear that as time progresses $(t>0.4)$ our numerical results approach those of the steady case discussed in the previous section. To know the transient behaviour, the variation of Nusselt number with time has also been considered and is depicted in Fig. 16. It is clear that as $R_{\mathrm{T}}$ increases, the system becomes more unsteady and shows sensitivity to the initial conditions. However, as time progresses a steady state is reached via a transient state.

\section{RESULTS}

The principal results of the foregoing linear and non-linear, rotating, double-diffusive convection in a sparsely packed, porous medium may be summarized as follows.
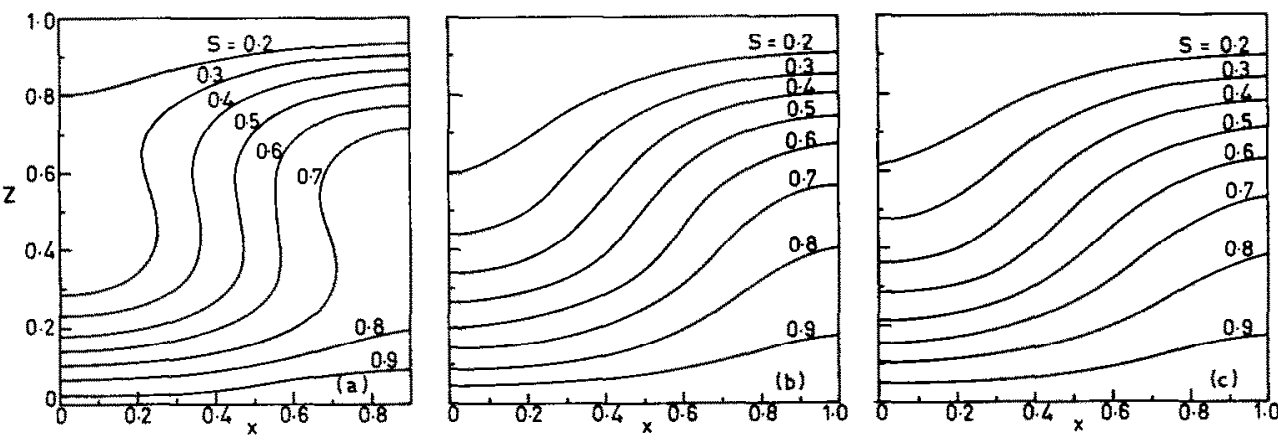

FiG. 14. Isohalines for $\tau=0.81, \sigma=0.2, T a=10^{4}, R_{\mathrm{s}}=10^{4}$ and $R_{\mathrm{T}}=1.1 R_{\mathrm{r}}^{\mathrm{c}}$ : (a) $P_{1}=10^{-2}$, (b) $P_{1}=10^{-3}$ and (c) $P_{1}=10^{-4}$. 

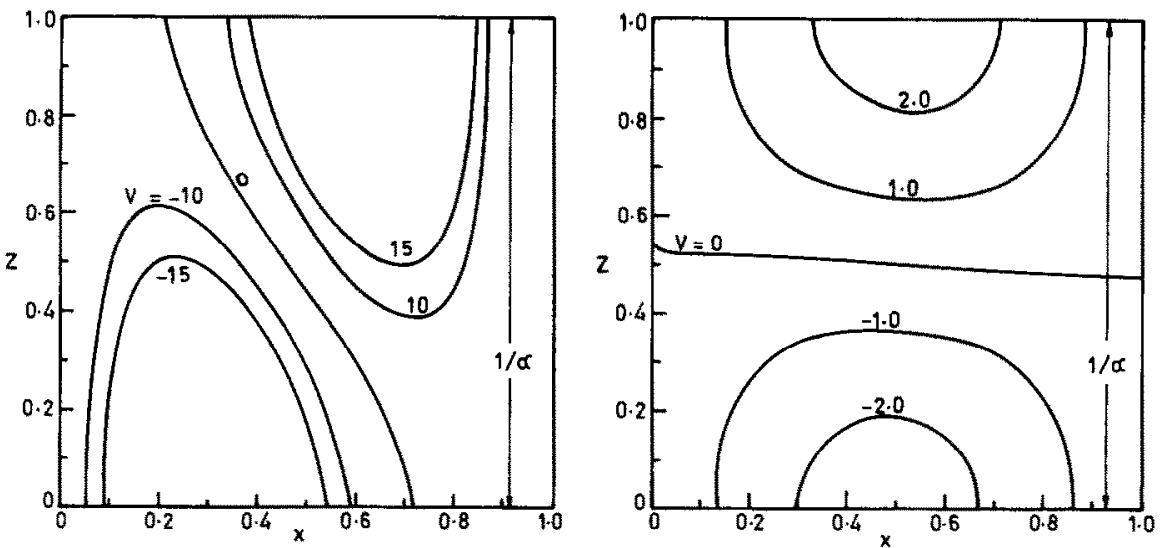

FiG. 15. Contour lines of the zonal velocity for $\tau=0.81, \sigma=0.2, T a=10^{4}, R_{\mathrm{s}}=10^{4}$ and $R_{\mathrm{T}}=1.1 R_{\mathrm{T}}^{\mathrm{c}}$; (a) $P_{1}=10^{-2} ;\left(\right.$ b) $P_{1}=10^{-3}$.

\section{Linear analysis}

(i) The critical wavenumber, in steady case, is independent of $R_{\mathrm{s}}$ and $\tau$ but depends on $T a$ and $P_{1}$. In other words, the cell pattern is influenced only by rotation and structure of the porous material and is independent of concentration gradient. From this, we conclude that the linear stability boundary is independent of $R_{\mathrm{s}}$ and $\tau$.

(ii) For $\tau<\sigma \eta<1$, a rotating porous layer can be destabilized by the addition of a bottom-heavy solute gradient.

(iii) For $\tau>$ on $<1$, we have shown the system which was stable in the absence of rotation becomes unstable in the presence of rotation. This destabilization is shown to associate with a monotonically increasing frequency.

(iv) The marginal stability of oscillatory modes occurs on a hyperboloid in $\left(R_{\mathrm{T}}, R_{\mathrm{s}}, R_{\mathrm{Ta}}\right)$ space but the space is very closely approximated by its planar asymptotes for any diffusivity ratio. It is

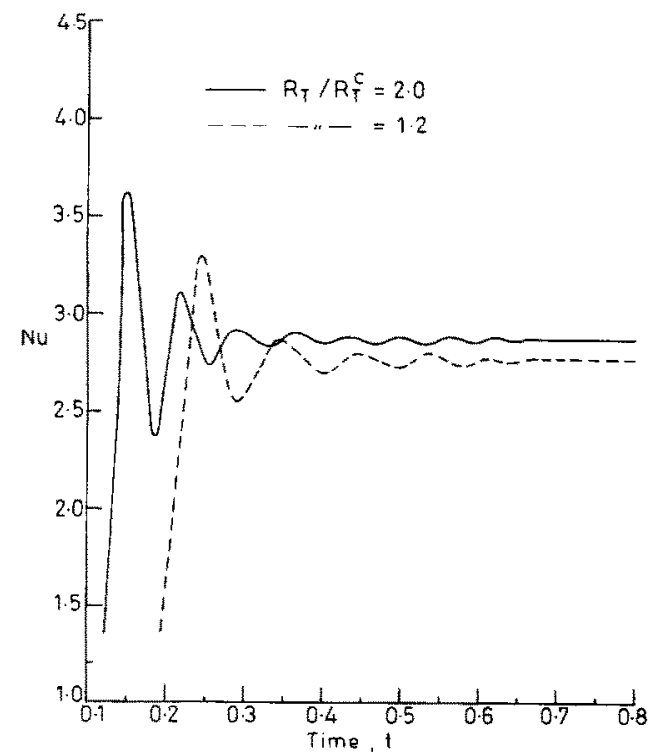

FIG. 16. Variation of Nusselt number with time $(t)$ for $T a=10^{2}, R_{\mathrm{s}}=10^{4}, \tau=0.32, P_{1}=10^{-2}$ and $\sigma=0.2$. found that the increase in $T a$ and decrease in $P_{1}$ is to stabilize the region of salt-finger and to increase the region of stability.

(v) Parameter differentiation provides result for the monotonic behaviour with respect to $R_{s}, T a, P_{1}$ and surface parameter such as Biot number.

\section{Non-linear analysis}

From the study of steady, finite-amplitude analysis we conclude that subcritical instabilities are possible and the range depend on $R_{\mathrm{s}}, \tau, P_{\mathrm{b}}, \sigma$ and $T a$. The heat and mass transport increase with an increase in $R_{\mathrm{T}}$, $R_{\mathrm{s}}$ and decrease with an increase in $T a$ and decrease in $P_{1}$ because the combined effect of rotation and porous parameter is to inhibit the onset of convection. Also decrease in $\tau$ and $\sigma$ increases $N u$ and $N u^{s}$. From the unsteady case, we see that the numerical results for large $t$ compare well with the steady case values. We conclude that the steady state emerges via a transient state of motion and an increase in $R_{\mathrm{T}}$ makes the system more unsteady.

Acknowledgements-This work was supported by the UGC under DSA programme. One of us (I.S.S) is grateful to the UGC for awarding a Senior Research Fellowship. The computational work in this paper was supported by the Department of Science and Technology, Government of India under the grant No. HCS/DST/1114/81.

Table 3. Time evolution of Nusselt numbers for $T a=10^{6}, R_{\mathrm{T}}=2 \times 10^{5}, P_{1}=10^{-2}$, $R_{\mathrm{s}}=10^{4}, \tau=0.32$ and $\sigma=0.2$

\begin{tabular}{ccc}
\hline$t$ & $\mathrm{Nu}$ & $\mathrm{Nu}$ \\
\hline 0.02 & 1.0003 & 1.0004 \\
0.04 & 1.0155 & 1.0266 \\
0.06 & 1.6512 & 2.1063 \\
0.08 & 3.0272 & 2.9212 \\
0.10 & 2.5194 & 2.8025 \\
0.14 & 2.6793 & 2.7959 \\
0.20 & 2.6718 & 3.0212 \\
0.30 & 2.6774 & 2.9579 \\
0.40 & 2.6776 & 2.9612 \\
0.50 & 2.6776 & 2.9615 \\
1.0 & 2.6776 & 2.9614 \\
2.0 & 2.6776 & 2.9614 \\
\hline
\end{tabular}




\section{REFERENCES}

1. A. Chakrabarti and A. S. Gupta, Nonlinear thermohaline convection in a rotating porous medium, Mech. Res. Commum. 8, 9 (1981).

2. D. A. Nield, Onset of thermohaline convection in a porous medium, Water Resources Res. 5, 553 (1968).

3. N. Rudraiah, P, K. Srimani and R. Friedrich, Finite amplitude thermohaline convection in a fluid saturated porous layer, Proc. 7 th Int. Heat Transfer Conference, Munich, F.R.G. (1982).

4. N. Rudraiah, P. K. Srimani and R. Friedrich, Finite amplitude convection in a two-component fluid saturated porous layer, Int. J. Heat Mass Transfer 25, 715 (1982).

5. R. W. Griffiths, Layered double-diffusive convection in porous media, J. Fluid Mech. 102, 221 (1981).

6. J. S. Turner, Buoyancy Effects in Fluids, Cambridge University Press, Cambridge (1979).

7. H. E. Huppert and J. S. Turner, Double-diffusive convection, J. Fluid Mech. 106, 299 (1981).

8. J. T. Stuart, On the nonlinear mechanics of hydrodynamic stability, J. Fluid Mech. 4, 1 (1958).

9. W. V. R. Malkus and G. Veronis, Finite amplitude cellular convection, J. Fluid Mech. 4, 225 (1958).

10. K. Vafai and C. L. Tien, Boundary and inertia effects on flow and heat transfer in porous media. Int. J. Heat Mass Transfer 24, 195 (1981).

11. N. Rudraiah, Nonlinear convection in a porous medium with convective acceleration and viscous force, Arab. $J$. Sci. Engng 9, 153 (1984).

12. D. D. Joseph, D. A. Nield and G. Papanicolaou, Nonlinear equation governing flow in a saturated porous medium, Water Resources Res. 18, 1049 (1982).

13. N. Rudraiah, P. K. Srimani and R. Friedrich, Convection in a rotating fluid saturated porous layer with convective acceleration, submitted for publication in Physica D (1985).

14. A. J. Pearlstein, Effect of rotation on the stability of a doubly diffusive fluid layer, $J$. Fluid Mech. 103, 389 (1981).

15. I. S. Shivakumara, N. Rudraiah and R. Narayanan, Further results on stability boundaries and a weak nonlinear analysis for double diffusive convection with rotation, Int. Commun. Heat Mass Transfer 12, 299 (1985).

16. H. Brenner, Rheology of two-phase systems, A. Rev. Fhid Mech, 2, 171 (1970).

17. P. G. Saffman, On the stability of laminar flow of a dusty gas, J. Fluid Mech. 13, 120 (1962)

18. T. M. Apostol, Mathematical Analysis. Addison-Wesley, Reading, MA (1957).

19. G. Veronis, Motions at subcritical values of the Rayleigh number in a rotating fluid, $J$. Fluid Mech 24, 545 (1966).

20. D. J. Acheson, 'Stable' density stratification as a catalyst for instability, J. Flud Mech. 96, 723 (1980).

21. R. Narayanan, Some differential inequalities for thermohaline convection in bounded containers of arbitrary shape, Int. J. Engng Sci. 22, 927 (1984).

22. B. Friedman, Principles and Techniques of Applied Mathematics. Wiley, New York (1956).

23. E. Knobloch and N. O. Weiss, Bifurcations in a model of magnetoconvection, Physica 9D, 379, (1983).

24. H. E. Huppert and D. A. Moore, Nonlinear double diffusive convection, J. Fluid Mech. 78, 821 (1976).

\section{APPENDIX}

Substituting $p=i p_{\mathrm{i}}$ in (13) and equating separately the real and imaginary parts to zero, we get

$$
\begin{aligned}
& \frac{p_{\mathrm{s}}^{4}}{\sigma}-p_{\mathrm{i}}^{2}\left[\delta_{1}+R_{\mathrm{Ta}}^{\prime}-R_{\mathrm{T}}^{\prime}+R_{\mathrm{s}}^{\prime}\right] \\
& \qquad+\tau \sigma \eta\left[\eta+\frac{R_{\mathrm{Ta}}^{\prime}}{\sigma \eta}-R_{\mathrm{T}}^{\prime}+\frac{R_{\mathrm{s}}^{\prime}}{\tau}\right]=0 \\
& p_{\mathrm{i}}^{2}=\frac{\sigma}{\delta_{2}}\left[\delta_{3}+R_{\mathrm{Ta}}^{\prime}(1+\tau)-R_{\mathrm{T}}^{\prime}(\sigma \eta+\tau)+R_{\mathrm{s}}^{\prime}(\sigma \eta+1)\right]
\end{aligned}
$$

where

$$
\begin{aligned}
& \delta_{1}=\sigma \eta^{2}+\frac{\tau}{\sigma}+2 \eta(1+\tau) \\
& \delta_{2}=1+\tau+2 \sigma \eta \\
& \delta_{3}=\sigma \eta^{2}+\sigma \eta^{2} \tau+2 \tau \eta .
\end{aligned}
$$

Together, (A1) and (A2) describe the curve

$$
\begin{aligned}
c_{1} R_{\mathrm{r}}^{\prime 2}+c_{2} R_{\mathrm{s}}^{\prime 2}+c_{3} R_{\mathrm{T}}^{\prime 2}+c_{4} R_{\mathrm{T}}^{\prime} R_{\mathrm{s}}^{\prime}+c_{9} R_{\mathrm{T}}^{\prime} R_{\mathrm{Ta}}^{\prime}+c_{6} R_{\mathrm{s}}^{\prime} R_{\mathrm{Ta}}^{\prime} \\
+c_{7} R_{\mathrm{T}}^{\prime}+c_{8} R_{\mathrm{s}}^{\prime}+c_{9} R_{\mathrm{Ta}}^{\prime}+c_{0}=0
\end{aligned}
$$

where the coefficients are

$$
\begin{aligned}
& c_{1}=-(\sigma \eta+\tau)(\sigma \eta+1) \\
& c_{2}=-(\sigma \eta+1)(\sigma \eta+\tau) \\
& c_{3}=-2 \sigma \eta(1+\tau) \\
& c_{4}=-2(\sigma \eta+1)(\sigma \eta+\tau)+\delta_{2}(1+\tau+2 \sigma \eta) \\
& c_{5}=-2(\sigma \eta+\tau)(1+\tau)+\delta_{2}(1+2 \tau+\sigma \eta) \\
& c_{6}=2(\sigma \eta+1)(1+\tau)-\delta_{2}(2+\tau+\sigma \eta) \\
& c_{7}=\left(\delta_{1} \delta_{2}-2 \delta_{3}\right)(\sigma \eta+\tau) \delta_{2} \delta_{3}-\delta_{2}^{2} \tau \eta \\
& c_{8}=-\left(\delta_{1} \delta_{2}-2 \delta_{3}\right)(\sigma \eta+1)-\delta_{2} \delta_{3}+\delta_{2}^{2} \eta \\
& c_{9}=-\left(\delta_{1} \delta_{2}-2 \delta_{3}\right)(1+\tau)-\delta_{2} \delta_{3}+\delta_{2}^{2} \tau \sigma^{-1} \\
& c_{0}=\delta_{3}^{2}-\delta_{1} \delta_{2} \delta_{3}+\delta_{2}^{2} \tau \eta^{2} .
\end{aligned}
$$

For oscillatory motions we also require $p_{i}^{2}>0$ in (Al) and (A2). This is true only if

$$
(\sigma \eta+\tau) R_{\mathrm{T}}^{\prime}-(\sigma \eta+1) R_{\mathrm{s}}^{\prime}-(1+\tau) R_{\mathrm{Ta}}^{\prime} \leqslant \delta_{3}
$$

and

$$
\delta_{1}-\left(R_{\mathrm{T}}^{\prime}-R_{\mathrm{s}}^{\prime}-R_{\mathrm{Ta}}^{\prime}\right)+M^{1 / 2} \geqslant 0
$$

must be satisfied on (A3), where

$M=R_{\mathrm{T}}^{\prime 2}+R_{\mathrm{s}}^{\prime 2}+R_{\mathrm{Ta}}^{\prime 2}+2\left(-R_{\mathrm{T}}^{\prime} R_{\mathrm{s}}^{\prime}-R_{\mathrm{T}}^{\prime} R_{\mathrm{Ta}}^{\prime}+R_{\mathrm{s}}^{\prime} R_{\mathrm{Ta}}^{\prime}\right)$

$-4 \tau \eta\left(-R_{\mathrm{T}}^{\prime}+\frac{R_{\mathrm{s}}^{\prime}}{\tau}+\frac{R_{\mathrm{Ta}}^{\prime}}{\sigma \eta}\right)+2 \delta_{1}\left(-R_{\mathrm{T}}^{\prime}+R_{\mathrm{s}}^{\prime}+R_{\mathrm{Tz}}^{\prime}\right)+\delta_{1}^{2}-4 \tau \eta^{2}$.

Whenever $\tau>0$ and $\sigma \eta<1$, (A3) is an hyperboloid of which inequalities (A4) select the appropriate branch: the conditions at which the most unstable oscillatory mode is marginally stable. 


\section{EFFET DE LA ROTATION SUR LA CONVECTION LINEAIRE ET NON LINEAIRE} DANS UN MILIEU POREUX DISPERSE FIXE

Résumé--La stabilité linéaire et non linéaire de la convection avec rotation dans un milieu poreux dispersé fixe est étudiẻe en considérant une équation non darcienne. Dans le cas d'une théorie linéaire les mouvements marginaux et superstables sont discutés. Dans le premier cas, on montre que l'effet du nombre de Taylor et du paramètre de porosité est de rendre le système plus stable. Dans le dernier cas, on montre que le gradient du soluté lourd en bas et la rotation déstabilisent le système sous certaines conditions. En traçant les frontières de stabilité dans le plan du nombre de Rayleigh, on montre que l'effet de la rotation et du paramètre de porosité est de diminuer la région d'instabilité. A partir de la théorie de l'opérateur selfadjoint, la variation de la valeur propre critique est étudiée avec les paramètres physiques et limites. Dans le cas de la théorie linéaire, on considère à la fois les cas stables et instables. Dans le cas instable, le comportement transitoire de la variation du nombre de Nusselt avec le temps est étudié en résolvant un modèle de Lorenz du septième ordre par une méthode numérique de Runge-Kutta-Gill. Des résultats intéressants sont obtenus en comparant ces résultats avec ceux du cas stable. Finalement, est étudié l'effet

du paramètre de porosité sur la fonction de courant, les isothermes, les isohalines et la vitesse.

\section{EINFLUSS DER ROTATION AUF DIE LINEARE UND NICHTLINEARE KONVEKTION IN EINEM HOCHPOROSEN MEDIUM}

Zusammenfassung-Die lineare und nichtlineare Stabilität der Konvektion in einem rotierenden, hochporösen Medium wurde mit Hilfe einer Nicht-Darcy-Gleichung untersucht. Fär den Fall der linearen Theorie wurden Grenz-Bewegungen und überstabile Bewegungen diskutiert. Im erstgenannten Fall zeigt sich, da $B$ der Einflu $B$ von Taylor-Zahl und Porosität das System stabiler macht. Im letzteren Fall zeigt sich, da $\beta$ Lösungsgradient und Rotation das System unter bestimmten Bedingungen destabilisieren. Beim Einzeichnen der Stabilitätsgrenzen in die Rayleigh-Zahlen-Ebene zeigt sich, daß Rotation und Porosität das Gebiet der Instabilitäten verkleinern. Durch Anwendung der Theorie des 'self-adjoint operators' wurde die Abhängigkeit des kritischen Eigenwertes von physikalischen und Rand-Parametern untersucht. Im Fall der nichtlinearen Theorie wurde das stationäre und instationäre Verhalten betrachtet. Im instationären Fall wurde das transiente Verhalten der Nusselt-Zahl untersucht. Dies geschah durch numerisches Lösen eines Lorenz-Modells 7. Ordnung unter Anwendung der Runge-Kutta-Gill Methode. Beim Vergleich dieser Ergebnisse mit denen des stationären Falles ergaben sich interessante Resultate. Zum Schluß wurde der Einfluß der Porosität auf Stromfunktion, Isothermen, Linien konstanten Salzgehaltes und ZonenGeschwindigkeit untersucht.

\section{ВЛИЯНИЕ ВРАЩЕНИЯ НА ЛИНЕЙНУЮ И НЕЛИНЕЙНУЮ ДИФФУЗИОННУЮ КОНВЕКЦИЮ В НЕПЛОТНОЙ ПОРИСТОЙ СРЕДЕ}

Аннотания-Исследуются линейная и нелинейная устойчивости конвекиии при вращении в неплотной пористой среде, течение в которой не подчиняется закону Дарси. В случае линейной теории рассматриваются маргинальное и сверхустойчивое движения. Показано, что в первом случае влияние числа Тейлора и коэффициента пористости должны сделать систему более стабильной. Во втором случае, однако, при определенных условиях градиент концентрации тяжелого растворенного вещества и вращение дестабилизируют систему. С помощью графика для границ устойчивости на плоскости чисел Рэлея показано, что влияние врашения и параметра пористости должно уменышать величину области неустойчивостей. Изменение критического собственного значения в зависимости от физических и граничных условий исследуется по теории самосопря женного оператора. В нелинейной теории рассматриваются случаи устойчивой и неустойчивой конвекции. В случае пеустойчивой конвекции переходиый режим, связанный с измснением во времени числа Нуссельта, исследуется путем решения модели Лоренца седьмого порядка методом Рунге-Кутта-Гилла. Полученные результаты сравниваются с данными для случая устойчивой конвекции. Кроме этого, изучается влияние параметра пористости на функиию тока, изотермы, изогалины и зональные скорости. 